\title{
Mechanisms of polymyxin resistance: acquired and intrinsic resistance in bacteria
}

\author{
Abiola O. Olaitan ${ }^{1}$, Serge Morand ${ }^{2}$ and Jean-Marc Rolain ${ }^{1 *}$ \\ ${ }^{1}$ Unité de Recherche sur les Maladies Infectieuses et Tropicales Emergentes CNRS-IRD UMR 6236, Méditerranée Infection, Faculté de Médecine et de \\ Pharmacie, Aix-Marseille-Université, Marseille, France \\ 2 Institut des Sciences de I'Evolution, CNRS-IRD-UM2, CC065, Université Montpellier 2, Montpellier, France
}

Edited by:

Faraj Barah, University of Kalamoon, Syria

Reviewed by:

Carlos F. Amábile-Cuevas, Fundación

Lusara, Mexico

Herbert P. Schweizer, Colorado State

University, USA

\section{*Correspondence:}

Jean-Marc Rolain, Unité de

Recherche sur les Maladies

Infectieuses et Tropicales

Émergentes (URMITE) CNRS-IRD

UMR 6236, Méditerranée Infection,

Faculté de Médecine et de

Pharmacie, Aix-Marseille-Université,

Marseille, France

e-mail: jean-marc.rolain@univ-amu.fr
Polymyxins are polycationic antimicrobial peptides that are currently the last-resort antibiotics for the treatment of multidrug-resistant, Gram-negative bacterial infections. The reintroduction of polymyxins for antimicrobial therapy has been followed by an increase in reports of resistance among Gram-negative bacteria. Some bacteria, such as Klebsiella pneumoniae, Pseudomonas aeruginosa, and Acinetobacter baumannii, develop resistance to polymyxins in a process referred to as acquired resistance, whereas other bacteria, such as Proteus spp., Serratia spp., and Burkholderia spp., are naturally resistant to these drugs. Reports of polymyxin resistance in clinical isolates have recently increased, including acquired and intrinsically resistant pathogens. This increase is considered a serious issue, prompting concern due to the low number of currently available effective antibiotics. This review summarizes current knowledge concerning the different strategies bacteria employ to resist the activities of polymyxins. Gram-negative bacteria employ several strategies to protect themselves from polymyxin antibiotics (polymyxin B and colistin), including a variety of lipopolysaccharide (LPS) modifications, such as modifications of lipid A with phosphoethanolamine and 4-amino-4-deoxy-L-arabinose, in addition to the use of efflux pumps, the formation of capsules and overexpression of the outer membrane protein $\mathrm{OprH}$, which are all effectively regulated at the molecular level. The increased understanding of these mechanisms is extremely vital and timely to facilitate studies of antimicrobial peptides and find new potential drugs targeting clinically relevant Gram-negative bacteria.

Keywords: lipopolysaccharides, lipid A, Enterobacteriaceae, non-fermentative bacilli, mutation, two-component systems, antibiotic resistance

\section{INTRODUCTION}

Recently, the rise in infections caused by multidrug-resistant (MDR) Gram-negative bacteria, especially the resistance to carbapenems most importantly observed in Pseudomonas aeruginosa, Acinetobacter baumannii, Klebsiella pneumoniae, and Escherichia coli, has led to the resuscitation of polymyxins (polymyxin B and colistin) worldwide as a last-resort treatment option (Stein and Raoult, 2002; Falagas and Michalopoulos, 2006; Biswas et al., 2012). Resistance to polymyxins by bacteria that are normally susceptible to these drugs has been reported (Johansen et al., 2008; Arduino et al., 2012; Mammina et al., 2012). There are also reports of increases in infections caused by naturally polymyxin-resistant bacteria, such as Proteus, Providencia, Morganella, and Serratia (Hayakawa et al., 2012; Merkier et al., 2013; Samonis et al., 2014).

Bacteria employ several means to protect themselves from adverse environmental stimuli, including exposure to cationic antimicrobial peptides, such as polymyxin B and colistin. These strategies include alterations of their lipopolysaccharides (LPSs), which have overall negative charges and are the initial targets of polymyxins (Moffatt et al., 2010). Such alterations can be achieved by covalent modifications of the lipid A moiety of LPS through the addition of phosphoethanolamine (PEtN) and 4-amino-4-deoxy-L-arabinose (L-Ara4N), deacylation, hydroxylation and palmitoylation by pagP (palmitoylation does not contribute to polymyxin resistance) (Ernst et al., 2001; Raetz et al., 2007). Other strategies include the utilization of an efflux pump and capsule formation (Campos et al., 2004; Padilla et al., 2010).

The most common LPS modification is the cationic substitution of the phosphate groups by L-Ara4N, which decreases the net negative charge of lipid A to 0 , and the second most common is the PEtN modification, which decreases the net charge from -1.5 to -1 (Nikaido, 2003). The L-Ara4N modification is the most effective of the two modifications due to the nature of the charge alteration. The resultant net positive charge of the modified LPS reduces its binding to polymyxins, leading to resistance.

The activation of two-component systems (TCSs) involving PhoP/PhoQ and PmrA/PmrB is triggered by environmental stimuli and specific mutations within the TCSs that result in their constitutive activation and subsequent overexpression of LPSmodifying genes (Gunn and Miller, 1996; Gunn et al., 2000; Trent et al., 2001b; Abraham and Kwon, 2009; Barrow and Kwon, 
2009; Miller et al., 2011). The activation of the PmrA/PmrB TCS leads to the upregulation of the pmrCAB and arnBCADTEF-pmrE (also called pmrHFIJKLM-ugd) operons that mediate the synthesis and transfer of PEtN and L-Ara4N, respectively, to lipid A (Gunn, 2001; Raetz et al., 2007; Yan et al., 2007). The PhoP/PhoQ TCS is known to contribute to polymyxin resistance by indirectly activating the $\mathrm{PmrA} / \mathrm{PmrB}$ TCS via $\mathrm{PmrD}$, except in various bacteria, such as E. coli (Kox et al., 2000; Kato et al., 2003; Winfield and Groisman, 2004). Once activated, the phosphorylated PmrA binds to the promoter region of the arnBCADTEF operon, increasing the recognition and binding of RNA polymerase and resulting in the upregulation of the operon (Wosten and Groisman, 1999).

There have been reports summarizing the mechanisms of resistance to polymyxins (Nation and Li, 2009; Falagas et al., 2010; Lim et al., 2010; Loutet and Valvano, 2011; Biswas et al., 2012; Cai et al., 2012); however, the lack of updated and comprehensive reports on the different mechanisms mediating or contributing to polymixin resistance including among the intrinsically-resistant bacteria has further necessitated this work.

\section{ACQUIRED RESISTANCE TO POLYMYXINS IN ENTEROBACTERIACEAE \\ ROLE OF LPS MODIFICATIONS \\ PmrA/PmrB and PhoP/PhoQ two-component system-mediated LPS modifications}

Salmonella enterica. Salmonella enterica serovar Typhimurium (S. Typhimurium) has been used as a model bacterium for most studies aimed at elucidating the mechanisms of resistance to cationic antimicrobial peptides (CAMPs), including polymyxins. Vaara et al. have observed that the LPSs of a polymyxin-resistant pmrA mutant of an $S$. Typhimurium strain contains 4-6 times more L-Ara4N in lipid A than the parent strain in addition to an increased level of PEtN (Vaara et al., 1981; Trent et al., 2001b). In polymyxin-resistant Salmonella, one principal mechanism underlying this modification, apart from external stimuli such as $\mathrm{Mg}^{2+}$, involves mutations in the PmrA/PmrB and PhoP/PhoQ TCSs. Mutations in these systems can cause their constitutive overexpression, resulting in the activation of the $\operatorname{arnBCADTEF}$ and pmrCAB operons and the modification of lipid A by L-Ara4N and PEtN, respectively (Figure 1) (Roland et al., 1993; Guo et al., 1997; Gunn et al., 1998; Zhou et al., 2001; Lee et al., 2004). Several mutations that activate the PmrA/PmrB TCS have been characterized in in vitro-selected mutant polymyxin-resistant $S$. Typhimurium (Table 1). Roland et al. have reported a missense mutation in pmrA, resulting in an amino acid substitution of R81H (Roland et al., 1993). An extensive genetic analysis of pmrA/pmrB of spontaneous colistin-resistant mutants has further identified numerous mutations, including a total of 27 independent missense mutations in the $p m r A$ and $p m r B$ genes that all result in the elevated expression of $\operatorname{arnB}$ ( $p m r H$ ) (Sun et al., 2009). The minimum inhibitory concentrations (MICs) of colistin for the spontaneous mutants reported range from 0.25 to $4.4 \mathrm{mg} / \mathrm{L}$, with most mutants displaying a 20-30-fold MIC increase. For PmrA, all mutations occurred in the phosphate receiver domain, while mutations occurred in 4 out of the 6 predicted domains in PmrB (Figure 2A). The histidine kinase gene $p m r B$ seems to be the more common site for bacterial mutations compared to the response regulator gene pmrA. However, not all missense mutations in $p m r A / p m r B$ result in their constitutive activation and polymyxin resistance. Some non-polymyxin resistance-causing missense mutations have been observed in the $p m r A / p m r B$ of various Salmonella serotypes (Agerso et al., 2012).

It has been observed that up to $88 \%$ of the $4^{\prime}$-phosphate of lipid A can be extensively modified with L-Ara4N in pmrA mutants of $S$. Typhimurium (Helander et al., 1994). Generally, L-Ara4N modifies the $4^{\prime}$-phosphate, while PEtN modifies the 1phosphate; however, in some cases, either one or two L-Ara4N and/or PEtN can be added to the 4'-phosphate and 1-phosphate of lipid A (Zhou et al., 2001; Lee et al., 2004). These two modifications are mediated by the arnBCADTEF operon and pmrC (also called eptA), respectively. Additionally, the phosphorylated heptose-I residue (LPS core) can be further modified with PEtN by the cptA gene (Figure 1) (Tamayo et al., 2005a). arnBCADTEF (also referred to as the $p m r F$ or $\operatorname{arnT}$ operon), pmrC and cptA are all regulated by pmrA in Salmonella (Tamayo et al., 2005b). The cationic modifications of these phosphate groups in LPSs are responsible for their reduced binding to polymyxins.

With regard to polymyxin resistance, the modification of LPSs by L-Ara4N confers a higher level of resistance than PEtN modifications (Tamayo et al., 2005a). Therefore, in terms of LPS modification-mediated polymyxin resistance in $S$. Typhimurium, the $\operatorname{arnT}$ operon is the most important, followed by $p m r C$ and cptA.

Furthermore, the 1-phosphate of lipid A can be phosphorylated to form 1-diphosphate (1-PP) by LpxT (Jones et al., 2008; Touze et al., 2008). This alteration increases the net negative charge of lipid A and reduces the efficiency of the PEtN modification (Herrera et al., 2010). These characteristic processes can increase the overall sensitivity of $S$. Typhimurium to polymyxins. However, LpxT activity is inhibited by the activation of pmra (Herrera et al., 2010) via PmrR (Pmr regulator) in S. Typhimurium, which further enhances resistance to polymyxins (Figure 1) (Kato et al., 2012). The biology of these genes (lpxT and $p m r R$ ) with respect to polymyxin resistance has yet to be fully elucidated, but it is highly probable that they may have a direct impact on this resistance considering their involvement in altering the overall charges of LPSs.

In Salmonella, the $R$-3-hydroxymyristate at position 3 of lipid A can be removed (deacylated) by pagL, which itself is activated by PhoP (Trent et al., 2001a). PagL is normally latent due to its inhibition by L-Ara4N and PEtN modifications of lipid A (Kawasaki et al., 2005, 2007), but the PagL-mediated deacylation of lipid A can occur in strains that are unable to modify this lipid A with either L-Ara4N or PEtN. Consequently, in such strains, the PagLdependent deacylation of this lipid A increases polymyxin resistance (Kawasaki et al., 2007). This finding implies that the various forms of lipid A modifications, particularly L-Ara4N and PEtN modifications and deacylation, may be compensatory to each other in certain bacteria, such as Salmonella. This relationship further depicts the complexity and intricacy of the interactions among the various mechanisms that mediate bacterial resistance to antimicrobial peptides, including polymyxins. 


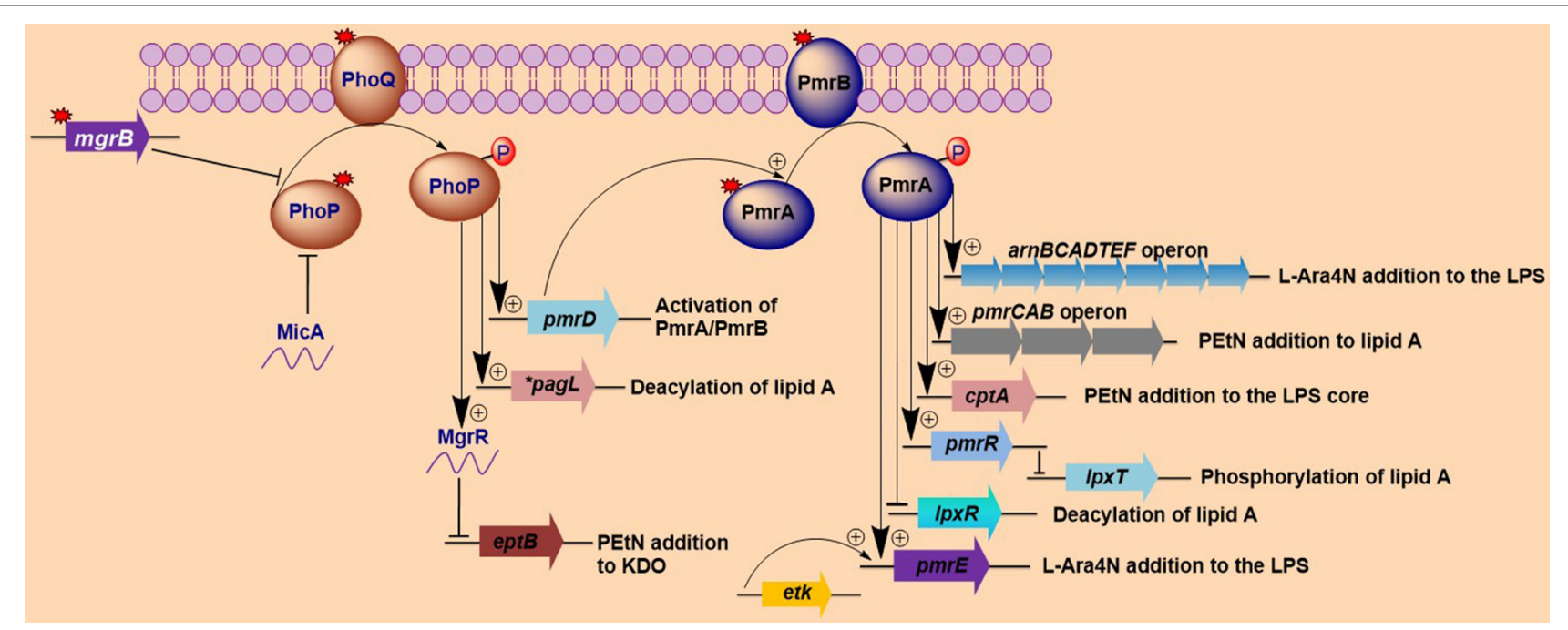

FIGURE 1 | Activation of lipopolysaccharide-modifying genes involved in polymyxin resistance in Gram-negative bacteria. Both MgrB and MicA (in Escherichia coli) exert negative feedback on the phoP/phoQ regulatory system, while mutations (denoted by red-colored star symbols) in $\mathrm{mgrB}$ or phoP/phoQ typically lead to the constitutive induction of the phoP/phoO two-component system. The activation of this two-component system (phoP/phoQ) activates pagL (which deacylates lipid A in Salmonella) and $p m r D$ (which in turn activates $p m r A$ ) and represses eptB via the activation of MgrR, with the resultant lipopolysaccharide (LPS) modifications all participating in the mediation of polymyxin resistance. Additionally, the phoP/phoQ regulatory system can directly activates the arnBCADTEFoperon in some bacteria such as Klebsiella pneumoniae. The repression of eptB prevents the modification of 3-deoxy-D-manno-oct-2-ulosonic acid (Kdo) with phosphoethanolamine (PEtN). The pmrA/pmrB two-component system is activated via $p m r D$ (which is activated by phoP) or through mutations in the pmrA/pmrB genes. Once induced, the phosphorylated pmrA activates the arnBCADTEF and pmrE genes, which collectively modify LPSs with 4-amino-4-deoxy-L-arabinose (L-Ara4N). The lipid A and the LPS core are further modified with PEtN by the pmrCAB operon and $c p t A$, respectively. Additional pmrA-activated genes include $p m r R$, which represses I $p x T$ (that phosphorylates lipid A) upon activation and IpxR gene (which deacylates lipid A). Lastly, etK can additionally phosphorylate the pmrE gene. The findings illustrated here are limited to modifications that have been shown to affect sensitivity to polymyxins. * pagL has only been found in Salmonella.
Some genes that have been implicated to be involved in polymyxin resistance and are independent of the PmrA/PmrB and PhoP/PhoQ genes include $r p o N$, which is an alternative sigma factor known to activate the transcription of several genes (Kazmierczak et al., 2005). S. Typhimurium with inactivated rpoN displayed polymyxin resistance by about two-fold, independent of $p m r A$, possibly due to the downregulation of polymyxin resistance-related gene(s) that are rpoN-regulated (Barchiesi et al., 2009).

The clinical relevance of polymyxin resistance in Salmonella currently seems to be non-existent, which is possibly because polymyxins are not currently used for treating infections caused by this bacterium. However, in vivo colistin resistance has been observed in animals such as pigs and poultry birds (EMEA, 2002; de et al., 2012; Morales et al., 2012; Kempf et al., 2013; Quesada et al., 2014), and such strains can be horizontally transmitted to humans.

Klebsiella pneumoniae. There have been a considerable number of studies aiming to elucidate polymyxin resistance in K. pneumoniae. In a genetically uncharacterized polymyxin-resistant strain of K. pneumoniae, the phosphate groups of lipid A have been observed to contain five times more L-Ara4N than the susceptible strain (Helander et al., 1996). This altered outer membrane composition is known to lower the negative charge of the outer membrane of K. pneumoniae (Velkov et al., 2013a), leading to the reduced interaction of this membrane with polymyxins. A molecular characterization of the structural alterations of LPSs in K. pneumoniae with regard to polymyxin resistance has similarly shown the involvement of phoP/phoQ and $p m r A / p m r B$ (Cheng et al., 2010). It has been observed that the phoP/phoQ and pmrA/pmrB systems are upregulated in K. pneumoniae exposed to polymyxins (Kim et al., 2014a), indicating that these systems are involved in polymyxin resistance in this bacterium. The constitutive activation of the $p m r A / p m r B$ system can also be caused by missense mutations in $p m r A$ or $p m r B$, leading to the subsequent upregulation of $p m r C$ and the arnBCADTEF operon, resulting in the synthesis and addition of PEtN and L-Ara4N, respectively, to lipid A, as shown in Figure 1. Recently, various occurrences of such mutations have been identified in both the $p m r A$ and $p m r B$ genes of clinical and non-clinical isolates of colistin-resistant $K$. pneumoniae, as shown in Table $\mathbf{1}$ and Figure 2B (Cannatelli et al., 2014b; Jayol et al., 2014; Olaitan et al., 2014b). In all the studies, the MIC range of colistin has been reported to be $3-$ $16 \mathrm{mg} / \mathrm{L}$ as shown by an Etest. Interestingly, similar mutations in the same loci have also been observed in colistin-resistant Salmonella and Enterobacter aerogenes (with a colistin MIC of $32 \mathrm{mg} / \mathrm{L}$ ) (Sun et al., 2009; Diene et al., 2013). However, several synonymous mutations that are not responsible for resistance have also been observed in pmrA and $p m r B$. Similarly, possible mutations in $p h o Q$ genes that result in resistance have also been observed in colistin-resistant K. pneumoniae (Olaitan et al., 2014b). 
Table 1 | Mutations in two-component systems that result in their constitutive activations.

\begin{tabular}{|c|c|c|c|}
\hline Bacteria & Gene & Mutation in aa & References \\
\hline \multirow[t]{20}{*}{$\begin{array}{l}\text { Salmonella } \\
\text { enterica }\end{array}$} & \multirow[t]{3}{*}{ pmrA } & $\mathrm{R} 81 \mathrm{H}, \mathrm{R} 81 \mathrm{C}$ & $\begin{array}{l}\text { Roland et al., 1993; Sun } \\
\text { et al., } 2009\end{array}$ \\
\hline & & G15R & Sun et al., 2009 \\
\hline & & G53E，G53R & \\
\hline & \multirow[t]{17}{*}{$p m r B$} & L14S, L14F & \\
\hline & & L22P & \\
\hline & & S29R & \\
\hline & & T92A & \\
\hline & & $\mathrm{P} 940$ & \\
\hline & & E121A & \\
\hline & & $\mathrm{S} 124 \mathrm{P}$ & \\
\hline & & $\mathrm{N} 130 \mathrm{Y}$ & \\
\hline & & $\mathrm{T} 147 \mathrm{P}$ & \\
\hline & & $\mathrm{R} 155 \mathrm{P}$ & \\
\hline & & T156P, T156 M & \\
\hline & & V161M, V161L, & \\
\hline & & V161G & \\
\hline & & E166K & \\
\hline & & M186I & \\
\hline & & G206W, G206R & \\
\hline & & S305R & \\
\hline
\end{tabular}

\begin{tabular}{|c|c|c|c|}
\hline \multirow{9}{*}{$\begin{array}{l}\text { Klebsiella } \\
\text { pneumoniae }\end{array}$} & pmrA & G53C & Olaitan et al., 2014b \\
\hline & $p m r B$ & L82R & Cannatelli et al., 2014b \\
\hline & & T157P & Jayol et al., 2014 \\
\hline & & S85R & Olaitan et al., 2014b \\
\hline & & T140P & \\
\hline & & $\Delta \mathrm{R} 14$ & Choi and Ko, 2014 \\
\hline & & $\Delta Y 209$ & \\
\hline & & T157P & \\
\hline & & S208N & \\
\hline $\begin{array}{l}\text { Enterobacter } \\
\text { aerogenes }\end{array}$ & pmrA & G53C & Diene et al., 2013 \\
\hline \multirow{10}{*}{$\begin{array}{l}\text { Acinetobacter } \\
\text { baumannii }\end{array}$} & pmrA & $\mathrm{M} 12 \mathrm{I}$ & Arroyo et al., 2011 \\
\hline & & S119T & \\
\hline & & E8D & $\begin{array}{l}\text { Lesho et al., 2013; Rolain } \\
\text { et al., } 2013\end{array}$ \\
\hline & & $\mathrm{P} 102 \mathrm{H}$ & Adams et al., 2009 \\
\hline & $p m r B$ & $\mathrm{~T} 13 \mathrm{~N}$ & \\
\hline & & $\mathrm{A} 227 \mathrm{~V}$ & \\
\hline & & P233S, P233T & \\
\hline & & $\mathrm{A} 262 \mathrm{P}$ & \\
\hline & & I121F & Park et al., 2011 \\
\hline & & A183T & \\
\hline
\end{tabular}

(Continued)
Table 1 | Continued

\begin{tabular}{|c|c|c|c|}
\hline \multirow[t]{37}{*}{ Bacteria } & \multirow[t]{37}{*}{ Gene } & Mutation in aa & \multirow[t]{5}{*}{ References } \\
\hline & & A184V & \\
\hline & & P190S & \\
\hline & & T192I & \\
\hline & & Q228P & \\
\hline & & S14L & \multirow[t]{8}{*}{ Beceiro et al., 2011} \\
\hline & & L87F & \\
\hline & & M145K & \\
\hline & & $\mathrm{A} 227 \mathrm{~V}$ & \\
\hline & & P233S & \\
\hline & & N353Y & \\
\hline & & F387Y & \\
\hline & & S403F & \\
\hline & & P170L & \multirow[t]{2}{*}{ Pournaras et al., 2014} \\
\hline & & P233S & \\
\hline & & $\Delta$ A32-E35 & \multirow[t]{15}{*}{ Arroyo et al., 2011} \\
\hline & & D64V & \\
\hline & & A80V & \\
\hline & & $\Delta \mathrm{L} 160$ & \\
\hline & & P1700, P170L & \\
\hline & & L208F & \\
\hline & & $\mathrm{A} 226 \mathrm{~V}$ & \\
\hline & & $\mathrm{R} 231 \mathrm{~L}$ & \\
\hline & & $\mathrm{P} 233 \mathrm{~S}$ & \\
\hline & & T235। & \\
\hline & & N256l & \\
\hline & & R263P, R263C & \\
\hline & & $\mathrm{O} 277 \mathrm{H}$ & \\
\hline & & G315D & \\
\hline & & P377L & \\
\hline & & S17R & \multirow[t]{4}{*}{ Lesho et al., 2013} \\
\hline & & $\mathrm{Y} 116 \mathrm{H}$ & \\
\hline & & T232I & \\
\hline & & $\mathrm{R} 263 \mathrm{~L}$ & \\
\hline & & $\mathrm{A} 227 \mathrm{~V}$ & \multirow[t]{3}{*}{ Kim et al., 2014b } \\
\hline & & P233S & \\
\hline & & FrF26 & \\
\hline \multirow{7}{*}{$\begin{array}{l}\text { Pseudomonas } \\
\text { aeruginosa }\end{array}$} & pmrA & L1570 & Lee and Ko, 2014 \\
\hline & \multirow[t]{6}{*}{$p m r B$} & M292T & $\begin{array}{l}\text { Abraham and Kwon, } \\
2009\end{array}$ \\
\hline & & L2430 & Moskowitz et al., 2004 \\
\hline & & A248V & \\
\hline & & $\Delta \mathrm{D} 45$ & $\begin{array}{l}\text { Schurek et al., 2009; } \\
\text { Moskowitz et al., } 2012\end{array}$ \\
\hline & & L14P & Moskowitz et al., 2012 \\
\hline & & A54V & \\
\hline
\end{tabular}


Table 1 | Continued

\begin{tabular}{|c|c|c|c|}
\hline \multirow[t]{22}{*}{ Bacteria } & \multirow[t]{22}{*}{ Gene } & Mutation in aa & \multirow[t]{10}{*}{ References } \\
\hline & & $\mathrm{R} 57 \mathrm{H}$ & \\
\hline & & $\mathrm{R} 79 \mathrm{H}$ & \\
\hline & & $\mathrm{R} 135 \mathrm{O}$ & \\
\hline & & G188D & \\
\hline & & A248T & \\
\hline & & S257N & \\
\hline & & $\mathrm{R} 259 \mathrm{H}$ & \\
\hline & & M292I & \\
\hline & & P456S & \\
\hline & & V15I & Lee and Ko, 2014 \\
\hline & & M48L & \\
\hline & & A67T & \\
\hline & & D70N & \\
\hline & & L167P & \\
\hline & & $\mathrm{H} 340 \mathrm{R}$ & \\
\hline & & T343A & \\
\hline & & $\mathrm{A} 247 \mathrm{~T}$ & $\begin{array}{l}\text { Owusu-Anim and Kwon, } \\
2012\end{array}$ \\
\hline & & M292T & \\
\hline & & Y345H & \\
\hline & & V281I & Choi and Ko, 2014 \\
\hline & & F237L & \\
\hline \multirow[t]{5}{*}{ K. pneumoniae } & phoP & G385S & Olaitan et al., 2014b \\
\hline & & L26Q & \\
\hline & phoQ & L96P & \\
\hline & & L3480 & \\
\hline & & S174N & Choi and Ko, 2014 \\
\hline \multirow[t]{18}{*}{$P$. aeruginosa } & phod & V260G & $\begin{array}{l}\text { Owusu-Anim and Kwon, } \\
2012\end{array}$ \\
\hline & & $\mathrm{H} 223 \mathrm{R}$ & \\
\hline & & V152 trunc. & \\
\hline & & A143V & Lee and Ko, 2014 \\
\hline & & K1230 & \\
\hline & & $\begin{array}{l}\text { Fr } 1421-428, X \\
429\end{array}$ & Miller et al., 2011 \\
\hline & & $1421 X^{d}$ & \\
\hline & & $D 433 X^{d}$ & \\
\hline & & $\Delta$ L364-G365 & \\
\hline & & $\Delta \mathrm{L} 364-\mathrm{G} 365$ & \\
\hline & & $\begin{array}{l}\text { R444C } \\
\text { R6C }\end{array}$ & \\
\hline & & $\Delta$ L364-G365 & \\
\hline & & $\Delta$ V57-0332 & \\
\hline & & K123E & Choi and Ko, 2014 \\
\hline & & $\mathrm{R} 214 \mathrm{H}$ & \\
\hline & & V184G & \\
\hline & & Q133E & \\
\hline & & A207R & \\
\hline
\end{tabular}

(Continued)
Table 1 | Continued

\begin{tabular}{|c|c|c|c|}
\hline Bacteria & Gene & Mutation in aa & References \\
\hline & \multirow{4}{*}{ parR } & \multicolumn{2}{|l|}{ N104l } \\
\hline & & N24S & Choi and Ko, 2014 \\
\hline & & L18| & \\
\hline & & S24N & \\
\hline & \multirow{10}{*}{ pars } & M59l & Muller et al., 2011 \\
\hline & & E156K & \\
\hline & & L140 & \\
\hline & & V101M & \\
\hline & & L137P & \\
\hline & & Q232E & Choi and Ko, 2014 \\
\hline & & G361R & \\
\hline & & $\mathrm{V} 295 \mathrm{~L}^{\dagger}$ & Lee et al., 2014a \\
\hline & & $\mathrm{A} 296 \mathrm{P}^{\dagger}$ & \\
\hline & & H398R & \\
\hline & colR & D32N & Muller et al., 2011 \\
\hline & cols & A106V & Gutu et al., 2013 \\
\hline & cprS & $\mathrm{R} 241 \mathrm{C}$ & \\
\hline
\end{tabular}

aa, amino acid; $\Delta$, deletion; trunc., truncation; $F r$, frameshift mutation; $X^{d}$, premature termination of amino acid; ${ }^{\dagger}$ predicted not to affect protein's function according to SIFT (Sorting Intolerant From Tolerant) analysis.

One profound molecular mechanism that leads to the emergence of colistin resistance in $K$. pneumoniae that has recently been discovered is the mutation/inactivation of the $\mathrm{mgrB}$ gene (Cannatelli et al., 2013), which is a conserved gene of 141 nucleotides in length (the length varies in naturally colistinresistant Enterobacteriaceae) encoding a short, 47-amino acid transmembrane protein that exerts negative feedback on the $\mathrm{PhoP} / \mathrm{PhoQ}$ regulatory system. It has been proposed that MgrB accomplishes this feedback by inhibiting the kinase activity of PhoQ and/or stimulating its phosphatase activity, which subsequently suppresses PhoP phosphorylation, leading to the repression of PhoP-regulated genes (Figure 1) (Lippa and Goulian, 2009). In accordance with this proposed mechanism, the deletion of $m g r B$ in $E$. coli has been observed to result in the upregulation of PhoP-regulated genes (Lippa and Goulian, 2009).

Disruption of the $m g r B$ gene in $K$. pneumoniae has been identified to play a prominent role in polymyxin resistance in this bacterium. Various disruptions in $m g r B$ have recently been described in diverse clinical and non-clinical isolates of colistinresistant $K$. pneumoniae and $K$. oxytoca, including insertional inactivation by an IS5-like element and other insertion sequences (Figure 3A) (Cannatelli et al., 2013; López-Camacho et al., 2013; Gaibani et al., 2014; Olaitan et al., 2014b; Poirel et al., 2014). Additional alterations that have been reported in $m g r B$ include a non-sense mutation leading to the premature termination of the MgrB transmembrane protein and missense mutations resulting in amino acid substitutions (Figure 3B) (Cannatelli et al., 2014a; Olaitan et al., 2014b; Poirel et al., 2014). The range of MICs for colistin that has been reported in these isolates is 
A
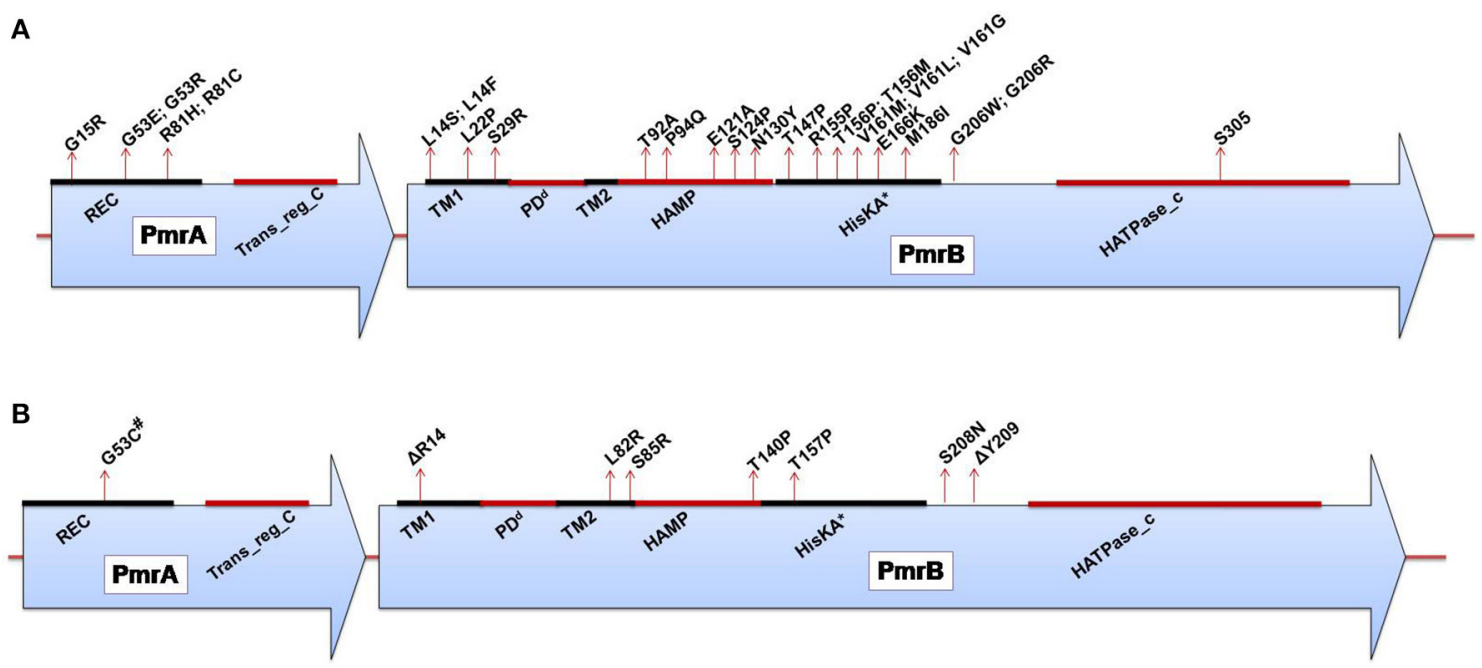

FIGURE 2 | (A) Domains of the PmrA/PmrB two-component system and positions of all mutations conferring polymyxin resistance to Salmonella enterica serovar Typhimurium. PmrA domains, cheY-homologous receiver domain [REC]; aa 1-112. Transcriptional regulatory protein, C-terminal domain [Trans_reg_C]; aa 145-216. PmrB domains, First transmembrane domain [TM1]; aa 13-35. dPeriplasmic domain [PD]; aa 35-66. Second transmembrane domain [TM2]; aa 66-88. Histidine kinases, adenylyl cyclases, methyl-binding proteins, and phosphatases [HAMP domain]; aa 89-141. Histidine kinase A (phosphoacceptor) domain [HisKA]; aa 142-202. Histidine kinase-like ATPases [HATPase_c]; aa 249-356. *HisKA, with the active site at $\mathrm{H} 148$ in PmrB of Salmonella enterica subsp. enterica serovar Typhimurium str. LT2 (GenBank accession no. AE006468). dPeriplasmic domain was not predicted in SMART but was assumed to be between the TM1 and TM2; aa, amino acid. (B) Domains of the PmrA/PmrB two-component system and positions of all mutations conferring polymyxin resistance to Klebsiella pneumoniae. PmrA domains, cheY-homologous receiver domain [REC]; aa 1-112. Transcriptional regulatory protein, C-terminal domain [Trans_reg_C]; aa 145-216.PmrB domains, First transmembrane domain [TM1]; aa 13-35. dPeriplasmic domain [PD]; aa 35-67. Second transmembrane domain [TM2]; aa 67-89. Histidine kinases, adenylyl cyclases, methyl-binding proteins, and phosphatases [HAMP domain]; aa 90-142. Histidine kinase A (phosphoacceptor) domain [HisKA]; aa 143-203. Histidine kinase-like ATPases [HATPase_c]; aa 250-358. *HisKA, with the active site at $\mathrm{H} 153$ in $\mathrm{PmrB}$ of $K$. pneumoniae subsp. pneumoniae MGH 78578 (GenBank accession no. CP000647). "Same mutation as that reported

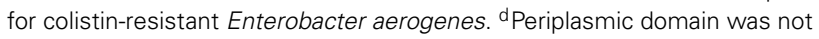
predicted in SMART but was assumed to be between TM1 and TM2; aa, amino acid; $\Delta$, deletion.

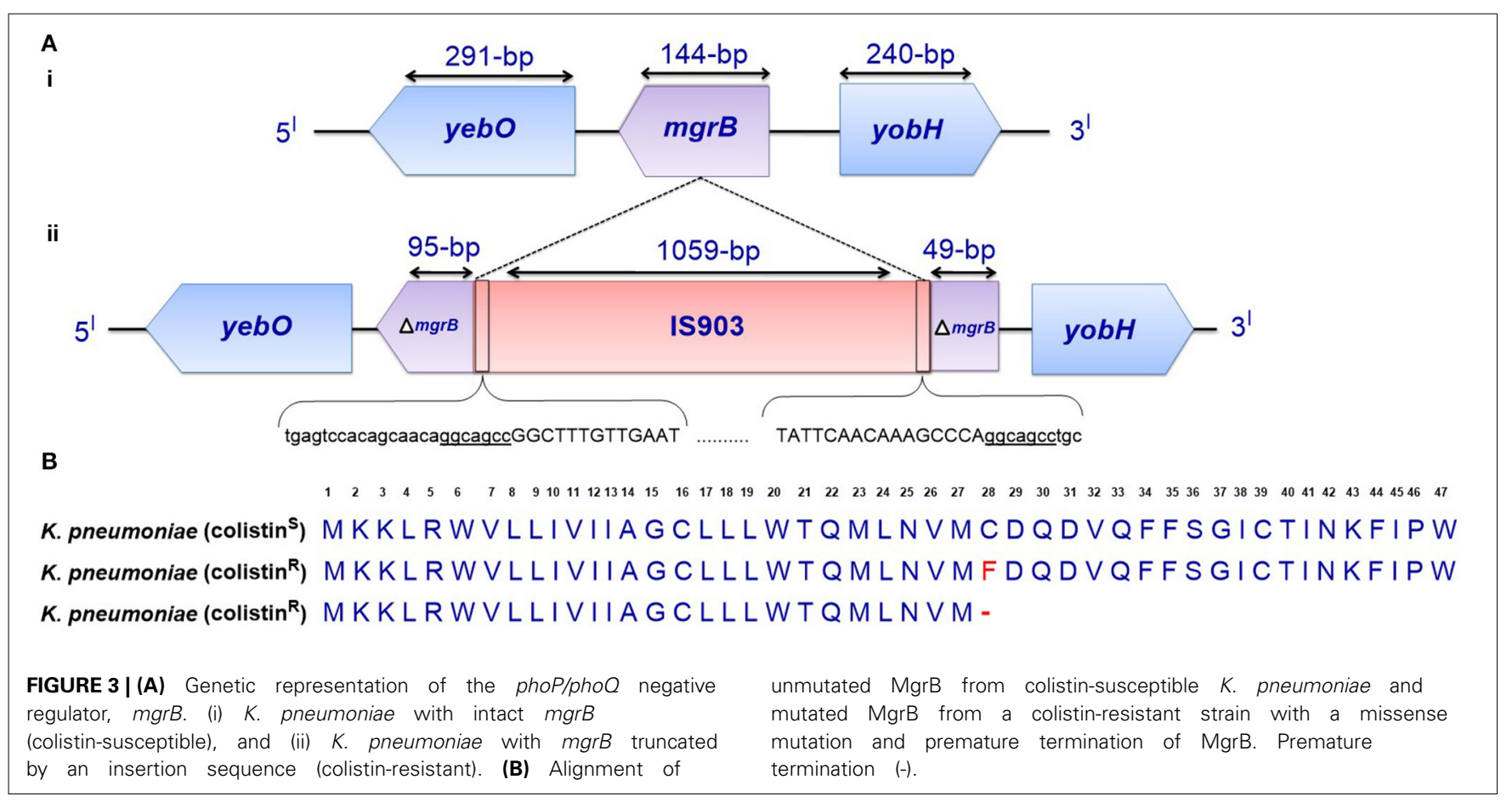


4-64 mg/L, as shown by Etest colistin strips. Several insertion sequences, such as those of the IS5-like, IS903B, IS1F-like and ISKpn14 elements belonging to several IS families (especially the IS5 family), have been observed to lead to the truncation of $m g r B$. An IS5D-like element initially localized on the plasmid has been observed to transposed to the chromosome and inactivate $m g r B$ (Cannatelli et al., 2014a; Olaitan et al., 2014b). Likewise, several amino acid substitutions, such as $\mathrm{C} 28 \mathrm{~F}$ (which has already been reported to affect PhoQ activity in E. coli (Lippa and Goulian, 2012), have been described in colistin-resistant K. pneumoniae (Cannatelli et al., 2014a; Olaitan et al., 2014b). Furthermore, a small deletion or the complete deletion of the $m g r B$ locus has been reported in various colistin-resistant strains (Cannatelli et al., 2014a; Olaitan et al., 2014b). Complementation studies carried out on some of these $m g r B$ mutant strains have resulted in the restoration of colistin sensitivity (Cannatelli et al., 2013, 2014a). This disruption of $m g r B$ can result in the upregulation of the arnBCADTEF operon, which adds L-Ara4N to lipid A (Cannatelli et al., 2013). The upregulation of the arnBCADTEF operon is attributed to phosphorylated PmrA, which is activated by $\mathrm{PmrD}$, which is in turn activated by the phosphorylation of PhoP resulting from the disruption of MgrB (Cheng et al., 2010; Kim et al., 2014a). Moreover, it has been demonstrated that activated PhoP can also directly activate the arnBCADTEF operon in K. pneumoniae independent of the PmrD- and PmrA-activated proteins in a phenomenon termed the feedforward connector loop (FCL) (Mitrophanov et al., 2008). We have recently observed that the mutation/inactivation of $m g r B$ accounts for greater colistin resistance among resistant $K$. pneumoniae compared with that resulting from TCS mutations ( $p m r A / p m r B$ or $p h o P / p h o Q)$ (Olaitan et al., 2014b). This observation demonstrates that $m g r B$ plays an important role in polymyxin resistance in K. pneumoniae.

A cross-regulatory interaction between PhoP/PhoQ and the regulator of the capsule synthesis (Rcs) phosphorelay system has been demonstrated in K. pneumoniae. Mutant $r c s B$ upregulates the expression of $p h o P / p h o Q$, implying that the Rcs system normally downregulates the phoP/phoQ system (Llobet et al., 2011). In light of this crosstalk between the two regulatory systems, it is plausible that a mutation in $\operatorname{rcs} B$ may play a role in polymyxin resistance via the upregulation of the $p h o P / p h o Q$ system in K. pneumoniae, although this mechanism remains to be demonstrated in polymyxin-resistant isolates.

Escherichia coli. In polymyxin-resistant E. coli, lipid A is typically modified with 2-aminoethanol and also with L-Ara4N (Nummila et al., 1995). The 2-aminoethanol molecule is added to the glycosidically linked diphosphate, whereas L-Ara4N is attached to the $4^{\prime}$-phosphate (Nummila et al., 1995). The importance of the $\mathrm{L}-\mathrm{Ara} 4 \mathrm{~N}$ modification for polymyxin resistance in $E$. coli has been further demonstrated in other studies (Yan et al., 2007).

As depicted in Figure 1, in addition to the phosphorylation of Ugd (or PmrE) and the arnT operon by the phosphorylated PmrA from the PmrA/PmrB TCS (Aguirre et al., 2000), Etk (a tyrosine-kinase) has been observed to phosphorylate Ugd, thereby participating in polymyxin resistance in E. coli (Lacour et al., 2006); the mutant E. coli strain displayed a reduced survival rate in the presence of polymyxin $\mathrm{B}$. The phosphorylation of the
Ugd protein (a UDP-glucose dehydrogenase) by Etk increases Ugd dehydrogenase activity. This elevated activity leads to an increase in the synthesis of UDP-glucuronic acid, which is the starting material for L-Ara4N synthesis by the arnT operon (Lacour et al., 2008). Interestingly, etk is expressed under conditions also known to activate the PmrA/PmrB system (Lacour et al., 2008). This finding indicates that, apart from the PmrA/PmrB system, polymyxin resistance via the modification of LPSs with L-Ara $4 \mathrm{~N}$ may be partially regulated by etk. Some studies have reported that Ugd is not directly activated by PmrA (Wosten and Groisman, 1999), but others have provided contrasting results (Aguirre et al., 2000). However, it is possible that etk is a principal activator of Ugd.

The 1-phosphate group of lipid A can be further modified by PEtN, albeit to a lesser extent (Kim et al., 2006). The addition of PEtN is mediated by $p m r C$ (also called eptA) and regulated by PmrA (Raetz et al., 2007). The lipid A of E. coli O157:H7 has been found to be distinctly modified by PEtN, most likely due to the increased activity of $p m r C$ in this serotype. This modification confers slight resistance to the cationic antimicrobial peptide PMBN (Kim et al., 2006). These findings demonstrate that alterations with L-Ara4N confer increased resistance to polymyxins compared with that of PEtN.

$m g r R$ is another genetic determinant that has been shown to mediate polymyxin resistance in $E$. coli. It is an Hfq-dependent sRNA that is one of the regulons known to be regulated by the $\mathrm{PhoP} / \mathrm{PhoQ}$ regulatory system in E. coli. $m g r R$ is conserved in other enteric bacterial genera, such as Salmonella, Citrobacter, Enterobacter, and Klebsiella. It has been described to negatively regulate $e p t B$, which mediates the modification of the outer Kdo (3-deoxy-D-manno-octulosonic acid) residues of LPSs with PEtN (Figure 1) (Reynolds et al., 2005; Raetz et al., 2007). $e p t B$ is indirectly influenced by the PhoP/PhoQ system (Moon and Gottesman, 2009). The modification of Kdo with PEtN reduces the net negative charges of LPSs, eventually leading to polymyxin resistance (Moon and Gottesman, 2009). It has been observed that the deletion of $m g r R$ in E. coli increases resistance to polymyxin B (Moon and Gottesman, 2009) as a result of the increased PEtN modification of Kdo. The expression of the PhoP/PhoQ TCS itself has been observed to be regulated by an Hfq-dependent sRNA termed MicA in E. coli and has been shown to repress the expression of PhoP (Coornaert et al., 2010). This process controls several PhoP-regulated genes, including MgrR. Thus, it is possible that MicA can also influence polymyxin resistance, although this influence has yet to be demonstrated.

It has recently been reported that cross-talk between QseB/QseC (quorum-sensing regulatory proteins) and PmrA/PmrB exists in E. coli. PmrB activates QseB in E. coli lacking QseC, and PmrA also directly regulates the transcription of QseB/QseC (Guckes et al., 2013). This interaction raises the possibility of cross-talk among other TCSs, which may influence the PhoP/PhoQ and/or PmrA/PmrB TCSs with respect to bacterial resistance to cationic antimicrobial peptides.

\section{Underacylation of lipid $A$ and its effect on polymyxin resistance} Salmonella enterica. The importance of the myristoylation of lipid A (a late secondary acylation) with regard to polymyxin resistance has been highlighted in Salmonella. Salmonella msbB 
strains (lp $x M$ mutants) that produce predominantly pentaacylated lipid A (underacylated) and are unable to add the myristoyl group to this lipid A display sensitivity to polymyxin (Tran et al., 2005; Murray et al., 2007). This sensitivity occurs because the myristoylation of lipid A is essential for the L-Ara4N modification of the phosphate groups of lipid A (Tran et al., 2005). Nonetheless, a mutation in $p m r A$ can still confer polymyxin resistance to a Salmonella lp $x M$ mutant as a result of an alternative lipid A modification, such as that involving PEtN, although this resistance is lower than that conferred by the addition of L-Ara4N. Therefore, the secondary acylation of lipid A is important for the addition of L-Ara4N (but not PEtN) to the phosphate groups of this lipid and for resistance to polymyxins.

Klebsiella pneumoniae. In K. pneumoniae, the lpxM gene also encodes the enzyme involved in the addition of the myristoyl group to lipid A, which results in the formation of hexa-acylated lipid A. An lpxM mutant of $K$. pneumoniae that produces predominantly penta-acylated lipid $\mathrm{A}$ has been found to be 8- to 16-fold more sensitive to both polymyxin B and colistin than the wild type with hexa-acylated lipid A (Clements et al., 2007). Velkov et al. have shown that the LPSs of the K. pneumoniae lpxM mutant strain with a colistin MIC of $0.25 \mathrm{mg} / \mathrm{L}$ display a four-fold higher binding affinity to polymyxins compared to the wild-type strain, with an MIC of $4 \mathrm{mg} / \mathrm{L}$ (Velkov et al., 2013b).

Similarly, a greater degree of acylation has been observed in polymyxin-resistant K. pneumoniae by Helander et al. (1996). Similar to Salmonella, underacylation has been reported to play an important role in polymyxin resistance in $K$. pneumoniae, likely due to the inability of underacylated K. pneumoniae to perform the L-Ara4N modification of lipid $\mathrm{A}$, as has been noted in other enteric bacteria (Tran et al., 2005). It has been alternatively proposed that the increased susceptibility of underacylated $K$. pneumoniae, which results in fewer acyl chains, leads to the enhanced insertion of the polar heads or the fatty acid tails of polymyxins into membranes and thus a higher binding affinity of lipid A to these antibiotics (Clements et al., 2007; Velkov et al., 2013b).

Escherichia coli. The myristoylation of lipid A by lpxM resulting in a hexa-acylated lipid A is likewise important for the addition of L-Ara4N to the lipid A of E. coli. Therefore, a loss of lipid A myristoylation in an $l p x M$ mutant results in a lack of L-Ara4N modification and a subsequent decrease in polymyxin resistance (Tran et al., 2005).

\section{Other LPS modifications mediating polymyxin resistance}

The removal of the $3^{\prime}$-acyloxyacyl residue from lipid $A$ has been observed in $S$. Typhimurium. This removal is mediated by $l p x R$, which is a hydrolase gene that encodes $3^{\prime}$-O-deacylase and has been shown to be present in other Gram-negative bacteria. $l p x R$ is usually latent because its expression is inhibited by PmrA/PmrBregulated lipid A modifications (Reynolds et al., 2006). The removal of 3'-acyloxyacyl results in the attachment of a reduced number of acyl groups to lipid A, and this alteration has been demonstrated to contribute to polymyxin resistance (Helander et al., 1996; Tran et al., 2005; Clements et al., 2007; Murray et al.,
$2007)$. The activation of $l p x R$ may play a role in polymyxin resistance, but the contribution of $l p x R$ to this resistance has not yet been demonstrated.

Recently, genomic analysis of colistin-resistant K. pneumoniae isolates has revealed that these isolates harbor eight distinct mutations compared to susceptible isolates that have been found in both non-coding and coding regions. Mutations have been detected in genes encoding a microcin transporter, a putative membrane protein, a putative transport protein and the methyl viologen-resistance protein SmvA (Snitkin et al., 2012), and the isolates display a colistin MIC range of $4-128 \mathrm{mg} / \mathrm{L}$. Another study involving a comparative genomic analysis of two colistin-resistant $K$. pneumoniae strains identified nonsynonymous mutations in the waaL, $r f b A$, and vacJ genes. All three of these genes are known to be involved in the biosynthesis of outer membrane proteins (Sassera et al., 2013). However, it is not yet known if these genes or their mutations contribute to colistin resistance. Furthermore, Salmonella enterica with a mutation in waaP (a gene encoding a protein involved in the phosphorylation of the heptose residue of the LPS inner core) has been shown to display a higher susceptibility to polymyxins (Yethon et al., 2000).

Overall, the activities of most of these genes are outer membrane-related, and any gene that acts to undermine the integrity of the bacterial outer membrane may play a role in polymyxin resistance (Snitkin et al., 2012). Therefore, some of these aforementioned genes need to be further scrutinized.

\section{ROLE OF CAPSULE IN POLYMYXIN RESISTANCE}

It has been reported that $K$. pneumoniae is able to shed capsular polysaccharides (CPSs) from its surface. The released CPSs are able to trap or bind to polymyxins, thereby reducing the quantity of drug that reaches the bacterial cell surface, leading to increased polymyxin resistance (Llobet et al., 2008). The underlying mechanism is attributed to electrostatic interactions between the cationic polymyxins and anionic CPSs.

It has been further observed that CPSs play a direct role in resistance to antimicrobial peptides, including polymyxin $\mathrm{B}$, by reducing their interactions with the bacterial outer membrane, mainly through the upregulation of capsular biosynthesis genes. CPS genes are further dependent on a certain CPS biosynthesis threshold for resistance (Campos et al., 2004). However, the role of the capsule in mediating polymyxin resistance remains under debate. Various studies have reported that the capsule does not contribute to resistance against antimicrobial peptides, including polymyxin B (Weiss et al., 1982; Clements et al., 2007). Interestingly, the ugd gene plays a dual role in CPS and L-Ara4N biosyntheses (Lacour et al., 2006), and its phosphorylation serves as a bridge between capsular synthesis and polymyxin resistance (Lacour et al., 2008).

\section{ROLE OF EFFLUX PUMP IN POLYMYXIN RESISTANCE}

A few studies have shown that efflux pumps can also contribute to polymyxin resistance. The efflux pumps that have been studied include AcrAB and KpnEF. It has been observed that $K$. pneumoniae with a mutation in $a c r B$ is significantly more susceptible to polymyxin B than the wild-type strain and that 
polymyxin B is pumped out of $K$. pneumoniae in an energydependent manner (Padilla et al., 2010). Another study has reported that mutant KpnEF (a member of the small MDR efflux pump family) is more susceptible to several antibiotics, including colistin, compared with wild-type K. pneumoniae and that this mutant displays a two-fold reduction in the colistin MIC compared with the wild-type strain (Srinivasan and Rajamohan, 2013). Interestingly, the same $k p n E F$ mutant also displays an impairment in capsular synthesis (Srinivasan and Rajamohan, 2013).

\section{ACQUIRED RESISTANCE TO POLYMYXINS IN NON-FERMENTATIVE BACILLI}

\section{Acinetobacter baumannii}

Two primary mechanisms that provide colistin resistance have been described in A. baumannii to date. The first is the modification of the lipid A moiety of LPS with PEtN as a result of mutations in the $p m r A / p m r B$ TCS. The second is the complete loss of LPSs caused by either mutations or the insertional inactivation of lipid A biosynthesis genes (Moffatt et al., 2010, 2011).

\section{PmrCAB-mediated LPS modifications}

A. baumannii does not possess the genetic machinery required for L-Ara4N biosynthesis; however, it contains an ortholog of the pmrCAB operon that mediates the addition of PEtN to its lipid A (Adams et al., 2009). Similar to enteric bacteria, the PmrA/PmrB TCS has been shown to mediate resistance to colistin in this bacterium. Mutations in $p m r A$ and/or $p m r B$ have been widely observed to induce the constitutive expression of $p m r A$ (the cognate response regulator) and the subsequent autoregulation of the promoter region of the $p m r C A B$ operon, which leads to the modification of the $4^{\prime}$-phosphate or 1-phosphate group of lipid A with PEtN. Missense or small indel mutations in the pmr locus (primarily in $p m r B$ ) in both colistin-resistant clinical isolates (in vivo) and in vitro-selected isolates have been shown to result in colistin resistance in A. baumannii (Table 1) (Arroyo et al., 2011; Beceiro et al., 2011; Lesho et al., 2013; Rolain et al., 2013; Snitkin et al., 2013). Mutations have been reported in different domains of both PmrA and PmrB (Figure 4A) (Arroyo et al., 2011; Beceiro et al., 2011; Lesho et al., 2013; Rolain et al., 2013). Additionally, pmrB appears to be the most commonly mutated gene in A. baumannii, similar to enteric bacteria (Figure 4A). In most of these studies, the MICs of colistin have ranged from 4 to $256 \mathrm{mg} / \mathrm{L}$ and from 4 to $128 \mathrm{mg} / \mathrm{L}$ as shown by Etest and broth microdilution techniques, respectively.

The reversion from a resistant to a susceptible phenotype has been observed in some strains in which the mutant pmr strain acquires an additional mutation (a compensatory mutation) elsewhere in the pmr locus that counteracts the hyper-activation of the TCS caused by the first mutation (Adams et al., 2009; Snitkin et al., 2013). It is also possible that the mutant pmr gene may revert back to its unmutated form, thereby reversing the resistance phenotype (Snitkin et al., 2013). However, compensatory mutations have not been observed in all revertants, with some strains maintaining the mutation in $p m r B$. This finding indicates the possible involvement of other unknown gene(s) in addition to the $p m r A / p m r B$ locus in the mediation of colistin resistance and its reversion in some colistin-resistant strains. This idea is supported by the findings of Park et al., who did not detect any mutations in the $p m r A / p m r B$ of six colistin-resistant $A$. baumannii clones out of 30 resistant isolates analyzed (Park et al., 2011), indicating the existence of other possible candidate gene(s). The identification of these genes is crucial for improving current knowledge of polymyxin resistance.

Colistin resistance in A. baumannii can emerge independently in a susceptible strain, and some strains may have a higher tendency of becoming resistant (Lesho et al., 2013). In some cases, A. baumannii possesses an auxiliary $p m r C$-like gene (named eptA), and some strains can have more than one copy of this gene (Arroyo et al., 2011; Lesho et al., 2013). It has been shown that the expression of this additional pmrC-like gene markedly increases in colistin-resistant strains. Interestingly, it has also been found to be located closer to mobile elements (prophage or integrase), suggesting the possibility of its horizontal acquisition (Lesho et al., 2013). The role of this auxiliary phosphoethanolamine transferase-encoding gene in colistin resistance in A. baumannii is still unknown.

Other modifications. Phosphorylation with PEtN usually occurs at the $4^{\prime}$-phosphate or 1-phosphate group of lipid A in A. baumannii colistin-resistant strains. In addition to this modification, a unique glycosylation of lipid A with galactosamine (hexosamine) at the 1-phosphate group has been recently reported in resistant strains (Pelletier et al., 2013). This unique modification is similar to the L-Ara4N modification in colistinresistant enteric bacteria. Clinical isolates with both PEtN and galactosamine modifications have been reported, displaying colistin MICs of 1.5-48 mg/L as shown by a colistin Etest (Pelletier et al., 2013). The genetic basis underlying this modification remains to be determined. Furthermore, a predominance of hepta-acylated lipid A has been observed in colistin-resistant A. baumannii by Beceiro et al. (2011). This increase in lipid A acylation appears to be significant to colistin resistance, and the unique pattern of modifications (phosphorylation and glycosylation) observed in colistin-resistant A. baumannii is similar to that reported in enteric bacteria (Tran et al., 2005).

\section{Loss of LPS-mediated colistin resistance}

In colistin-resistant $A$. baumannii, mutations have been reported that involve a nucleotide substitution or deletion and an insertional inactivation with the insertion of ISAba11 element in the first three lipid A biosynthesis genes, namely $l p x A$, lp $x C$, and lp $x D$, resulting in the complete loss of LPSs (Moffatt et al., 2010, 2011). Resistant strains harboring such mutations display a $>128 \mathrm{mg} / \mathrm{L}$ colistin MIC with broth microdilution, and they lack LPSs because of their inability to synthesize lipid A. Recent analysis of polymyxin B-resistant A. baumannii isolates has further shown the presence of unique mutations in the $l p x C$ and $l p x D$ genes (Lean et al., 2014) in addition to the mutations found in the lps $B$ gene encoding a glycosyltransferase (involved in the biosynthesis of the LPS core) that have also been implicated in colistin resistance in A. baumannii (Hood et al., 2013; Lean et al., 2014). Because lipid A is the initial target of colistin, its absence results 


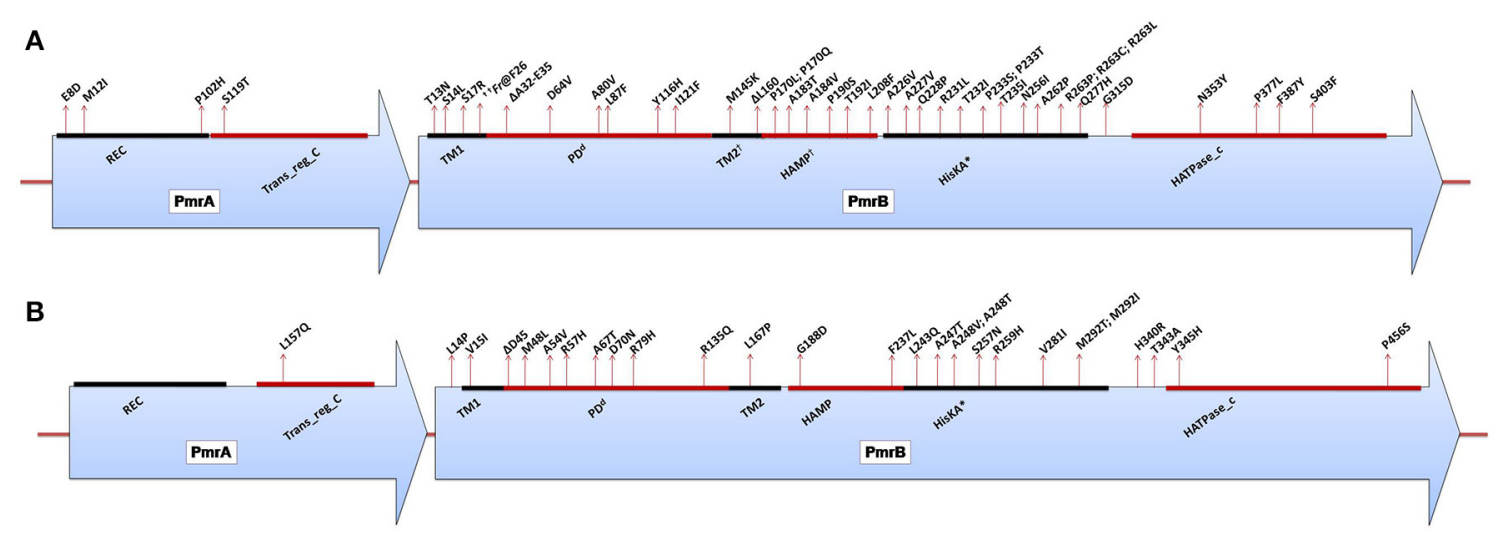

FIGURE 4 | (A) Domains of the PmrA/PmrB two-component system and positions of all mutations conferring polymyxin resistance to Acinetobacter baumannii. PmrA domains, cheY-homologous receiver domain [REC]; aa 2-112. Transcriptional regulatory protein, C-terminal domain [Trans_reg_C]; aa 150-221. PmrB domains, First transmembrane domain [TM1]; aa 10-29. dPeriplasmic domain [PD]; aa 29-142. †Second transmembrane domain [TM2]; aa 142-164. 'Histidine kinases, adenylyl cyclases, methyl-binding proteins, and phosphatases [HAMP domain]; aa 145-214. Histidine kinase A (phosphoacceptor) domain [HisKA]; aa 218-280. Histidine kinase-like ATPases [HATPase_c]; aa 326-437. *HisKA, with the active site at $\mathrm{H} 228$ in PmrB of Acinetobacter baumannii ATCC17978 (GenBank accession no. CP000521). dPeriplasmic domain was not predicted in SMART but was assumed to be between TM1 and TM2. ${ }^{\dagger}$ TM2 and HAMP overlapped based on SMART prediction. ${ }^{\dagger \dagger} \mathrm{Fr}$ denotes frameshift mutation; aa, amino acid. (B) Domains of the PmrA/PmrB two-component system and positions of all mutations conferring polymyxin resistance to Pseudomonas aeruginosa. PmrA domains, cheY-homologous receiver domain [REC]; aa 1-112. Transcriptional regulatory protein, C-terminal domain [Trans_reg_C]; aa 145-216. PmrB domains, First transmembrane domain [TM1]; aa 15-37. dPeriplasmic domain [PD]; aa 38-160. Second transmembrane domain [TM2]; aa 161-183. Histidine kinases, adenylyl cyclases, methyl-binding proteins, and phosphatases [HAMP domain]; aa 186-238. Histidine kinase A (phosphoacceptor) domain [HisKA]; aa 239-304. Histidine kinase-like ATPases [HATPase_c]; aa 348-459. *HisKA, with the active site at $\mathrm{H} 249$ in PmrB of Pseudomonas aeruginosa PAO1 (GenBank accession no. AE004091). dPeriplasmic domain was not predicted in SMART but was assumed to be between TM1 and TM2; aa, amino acid. in the loss of the colistin target site and a high level of resistance in A. baumannii.

Finally, transposon mutagenesis performed to elucidate the mechanisms underlying adaptive resistance to colistin has identified over 20 genes, most of which participate in processes that protect bacteria from osmotic stress, which likely occurs due to colistin exposure (Hood et al., 2013). Therefore, the expression of these genes stimulated by the syntheses of compatible solutes and the expression of proteases can attenuate such stress.

Colistin heteroresistance in A. baumannii. Colistin heteroresistance has been widely observed among A. baumannii clinical isolates at levels as high as 90\% (Li et al., 2006; Barin et al., 2013). These resistant subpopulations have been reported to significantly contribute to both the regrowth and increased development of colistin resistance ( $\mathrm{Li}$ et al., 2006). However, the underlying molecular mechanism involved in this phenomenon remains to be elucidated, and its understanding is critical due to the clinical significance of colistin heteroresistance.

Fitness cost of colistin resistance in A. baumannii. Development of colistin resistance in A. baumannii may incur fitness costs to this microorganism. Such costs have been observed in colistinresistant isolates both in vitro and in vivo and include growth retardation, impaired virulence, and substantially reduced clinical invasiveness (Fernandez-Reyes et al., 2009; Lopez-Rojas et al., 2011; Rolain et al., 2011; Pournaras et al., 2014). Furthermore, Hraiech et al. have reported that colistin-resistant A. baumannii possesses a reduced ability to cause infections, including systemic dissemination and lung damage, in a rat model with pneumonia (Hraiech et al., 2013). Some of these biological costs, such as the impairment of virulence, have been linked with the reduced expression of metabolic proteins and of the OmpA porin (involved in the virulence of Acinetobacter) (Lopez-Rojas et al., 2011). Proteomic analysis of in vitro-selected colistinresistant A. baumannii has revealed the differential expression of 35 proteins, the majority of which are downregulated in the resistant strain. These proteins include outer membrane proteins, chaperones, translation factors, and enzymes involved in metabolism (Fernandez-Reyes et al., 2009). Interestingly, some of these isolates incurring biological cost also harbor mutations in the $p m r A / p m r B$ TCS (Fernandez-Reyes et al., 2009; Lopez-Rojas et al., 2011; Rolain et al., 2011; Hraiech et al., 2013; Pournaras et al., 2014). Beceiro et al. have recently demonstrated significant biological costs (in virulence and fitness) in colistin-resistant A. baumannii $l_{p x}$ mutants (lacking LPSs) compared to colistinresistant $A$. baumannii pmrA/pmrB mutants (PEtN-modified LPSs) (Beceiro et al., 2014).

\section{Pseudomonas aeruginosa}

The mode of polymyxin resistance in $P$. aeruginosa is very similar to that observed in enteric bacteria. Unlike A. baumannii, $P$. aeruginosa has both the $p m r A / p m r B$ and $p h o P / p h o Q$ TCSs, each of which can separately regulate the arnBCADTEF operon (McPhee et al., 2003). To date, five TCSs have been described to play a role in polymyxin resistance in $P$. aeruginosa as follows: PmrA/PmrB (McPhee et al., 2003; Moskowitz et al., 2004, 2012; Abraham and Kwon, 2009), PhoP/PhoQ (Macfarlane et al., 1999; Barrow and Kwon, 2009; Schurek et al., 2009; Miller et al., 
2011), ParR/ParS (Fernández et al., 2010; Fernandez et al., 2012), ColR/ColS and CprR/CprS (Gutu et al., 2013).

\section{PmrA/PmrB and PhoP/PhoQ two-component system-mediated LPS modifications}

Several studies have shown that $P$. aeruginosa can develop resistance to polymyxins via the constitutive modification of its LPSs with L-Ara4N, which is stimulated by the $p m r A / p m r B$ and phoP/phoQ TCSs (Macfarlane et al., 1999, 2000; McPhee et al., 2003). The constitutive stimulation of these regulatory systems is typically (but not always) induced by mutations (Moskowitz et al., 2004, 2012). The synthesis and transfer of L-Ara4N to the lipid A moiety of LPSs are accomplished by the arnBCADTEF operon in $P$. aeruginosa, as has been observed in enteric bacteria.

For the $p m r A / p m r B$ TCS, several polymyxin-resistant $P$. aeruginosa strains harboring mutations in $p m r B$ have been reported (Table 1). These genetic alterations are known to lead to the modification of lipid A with L-Ara4N. Moreover, various resistant isolates harbor double mutations in $p m r B$, and these multimutant strains tend to display high levels of resistance to colistin (Moskowitz et al., 2012). For P. aeruginosa, only one study has reported a mutation in pmrA that may be responsible for resistance to date (Lee and Ko, 2014), while the rest of the mutations have been mainly localized to PmrB or generally distributed within the cognate regulators of TCSs (Figure 4B; Table 1). Mutations have been observed in different portions of the PmrB domain (Moskowitz et al., 2012; Lee and Ko, 2014), and mutations in the periplasmic and DHP (dimerization and phosphoacceptor) domains, particularly in the histidine box motif, appear to be more significant (Moskowitz et al., 2012), i.e., those close to the putative active histidine residue (H249) tend to evoke greater polymyxin resistance than those distant from this residue (Abraham and Kwon, 2009). In most of the P. aeruginosa pmrA/pmrB mutants studied, the MICs of colistin have commonly ranged from 4 to $>512 \mathrm{mg} / \mathrm{L}$, mostly performed by the broth microdilution method.

In terms of lipid A modifications in resistant bacteria, there appears to be an absolute relationship of colistin resistance and L-Ara4 $\mathrm{N}$ addition as well as a significant relationship of this resistance and the loss of secondary lauroyl chain hydroxylation (Miller et al., 2011; Moskowitz et al., 2012). There seems to be wide structural variation in lipid A in polymyxinresistant $P$. aeruginosa in terms of the presence of either pentaor hexa-acylated lipid A (Moskowitz et al., 2012) in contrast to enteric bacteria, in which a higher degree of acylation is necessary for the addition of L-Ara4N to lipid A (Tran et al., 2005).

Another TCS that has been extensively studied and that also confers resistance in $P$. aeruginosa upon activation is the phoP/phoQ TCS. Several mutations resulting in in-frame deletions, frameshifts or truncations of the $p h o Q$ gene that consequently activate the arnBCADTEF operon have been reported in resistant strains (Table 1) (Miller et al., 2011; Lee and Ko, 2014). However, not all non-synonymous mutations in phoQ result in the constitutive activation of the system (Miller et al., 2011). In contrast to Salmonella, polymyxin resistance caused by the activation of $p h o P / p h o Q$ is not dependent on $p m r A / p m r B$ in $P$. aeruginosa (Miller et al., 2011). The mutations responsible for PhoP/PhoQ activation appear to occur in $p h o Q$, whereas mutations in phoP tend to act as primary suppressors in susceptible strains possessing mutations in both genes (Miller et al., 2011). In most of the P. aeruginosa phoP/phoQ mutants, colistin MICs usually ranged from 8 to $>512 \mathrm{mg} / \mathrm{L}$ and were mostly performed by the broth dilution technique. It has been recently reported that mutations in both $p m r B$ and $p h o Q$ result in greater resistant to polymyxins compared to a single mutation in either of the two genes (Owusu-Anim and Kwon, 2012).

Reversions from polymyxin-resistant to polymyxinsusceptible phenotypes among TCS ( $p m r A / p m r B$ or $p h o P / p h o Q)$ mutants have been observed, and they can occur by the following mechanisms: the reversion of mutant $p m r B$ or $p h o Q$ back to the wild-type gene (loss of mutation), an additional mutation in phoP or even the truncation of $p h o Q$ due to a secondary mutation (Miller et al., 2011; Lee and Ko, 2014). However, there is a high possibility that there are other gene(s) that may act as secondary suppressors when altered because some susceptible strains harbor polymyxin-inducing mutations in $p h o Q$ with wild-type $p h o P$ (Miller et al., 2011).

\section{Other two-component systems}

The ColR/ColS and CprR/CprS TC regulatory systems have been reported to mediate resistance in $P$. aeruginosa either directly or indirectly. Mutations in these two systems have been observed to contribute to high levels of polymyxin resistance in phoQ mutant isolates (Gutu et al., 2013). From one viewpoint, the involvement of colR/colS and $c p r R / c p r S$ in polymyxin resistance could occur through an interaction with the $\mathrm{PhoP} / \mathrm{PhoQ}$ regulatory system that enhances $p h o \mathrm{Q}$ activity and influences L-Ara4N modifications, which could lead to high levels of resistance. This interaction could be initiated by the presence of mutations in colR/colS and cprR/cprS (Table 1). Alternatively, Gutu et al. have suggested that the colR/colS and $\operatorname{cprR} /$ cprS systems may regulate other unknown genes involved in polymyxin resistance in addition to mediating L-Ara4N modifications via mutant phoQ (Gutu et al., 2013). This finding indicates that the L-Ara4N modification alone may not be sufficient for the development of polymyxin resistance in P. aeruginosa.

Additionally, $c p r R / c p r S$ and another TCS (designated parR/parS) have been shown to be involved in adaptive resistance to polymyxins and other antimicrobial peptides. The cprR/cprS TCS participates in adaptive resistance by sensing various antimicrobial peptides, such as the synthetic peptide $\mathrm{CP} 28$ and polymyxins, and upregulating the arnBCADTEF operon (Fernandez et al., 2012). Meanwhile, parR/parS can cause the upregulation of the LPS modification operon under sub-inhibitory concentrations of polymyxins and other CAMPs, such as indolicidin but not CP28 (Fernández et al., 2010), demonstrating that the two TCSs are activated by different antimicrobial peptides besides polymyxins. Furthermore, mutations in either ParR or ParS have been observed to cause the constitutive expression of the arnBCADTEF operon independent of PmrA/PmrB, resulting in polymyxin resistance. Colistin MIC 
of $2 \mathrm{mg} / \mathrm{L}$ (by agar dilution method) was reported for the clinical strains of $P$. aeruginosa harboring mutations in parR/parS genes. This activity has been observed in addition to the mediation of other antibiotic-related genes in $P$. aeruginosa by ParR/ParS (Muller et al., 2011).

\section{Other polymyxin resistance determinants in P. aeruginosa}

It has been shown that the overexpression of the outer membrane protein OprH in either an $\mathrm{Mg}^{2+}$-deficient medium or in a mutant parent strain can result in polymyxin resistance (Young et al., 1992). This is because OprH is a basic protein that binds to divalent cation-binding sites of LPSs, making these sites unavailable for polymyxin binding. However, it has also been suggested that the overexpression of OprH alone is not sufficient for polymyxin resistance in P. aeruginosa (Young et al., 1992)

Using transposon mutagenesis, other genes that may play roles in polymyxin resistance have been identified. A total of 17 genes were identified to contribute to intrinsic resistance in $P$. aeruginos $a$ and thus confer supersusceptibility phenotype to polymyxin; the mutant strains showed between 1.5- and 3- fold MIC reduction to polymyxin B compared to the wild-type strain. Notably among these genes, galU, $l p t C$, wapR, and ssg participate in LPS biosynthesis-related functions (regulatory functions, metabolism, synthesis and transport) (Fernandez et al., 2013). Disruptions in any of these LPS-mediated genes have been reported to affect the permeability of the outer membrane or to hinder LPS modifications, causing $P$. aeruginosa to be more susceptible to polymyxins (Fernandez et al., 2013).

Other genes that have been recently described to possibly play roles in resistance/susceptibility include those that are able to influence LPS modifications via $p m r A / p m r B$ [including a TCS hybrid (PA2583), an arabinose efflux permease-encoding gene (PA5548), a lipoprotein-encoding gene (PA1199), and a hypothetical protein-encoding gene (PA2928)] as well as nonLPS-mediated genes [the response regulator eraR (PA1980), the glycosyl transferase $w b p Z$ (PA5447) and hypothetical proteinencoding genes (PA4541 and PA1938)]. All of these genes have been mapped against the PAO1 genome (Lee et al., 2014b). The mutant strains display between 0.5 - and 4- fold colistin MIC reduction compared to the colistin-resistant wild-type strain with the exception the $\mathrm{p} d x B$ mutant. The full characterization of these genes would improve the understanding of the complex mechanisms guiding polymyxin resistance in $P$. aeruginosa and help to determine whether these genes are part of the other alternative pathways that have been suggested to mediate colistin resistance in this bacterium (Lee et al., 2014a).

\section{INTRINSIC RESISTANCE TO POLYMYXINS IN BACTERIA}

There are a number of Gram-negative bacteria that are naturally resistant to polymyxins, including but not limited to the following: Proteus spp., Providencia spp., Morganella morganii (all three collectively referred to as the Proteeae tribe), Serratia spp., Edwardsiella tarda and Burkholderia cepacia complex (Muyembe et al., 1973; Rozalski et al., 1997; Loutet and Valvano, 2011; Biswas et al., 2012; Samonis et al., 2014). Most of the aforementioned naturally resistant bacteria have LPSs that are modified with L-Ara4N, which may explain their intrinsic resistance (Basu et al., 1986; Boll et al., 1994; Vinogradov et al., 2006).

\section{Proteus mirabilis}

The lipid A and Kdo residue of the LPSs of wild-type Proteus mirabilis are known to contain L-Ara4N (Sidorczyk et al., 1983; Boll et al., 1994), which is believed to contribute to the intrinsic resistance of this bacterium to polymyxins. On the other hand, polymyxin-susceptible mutants usually lack L-Ara4N in their LPSs, and those showing reduced resistance contain fewer L-Ara4N substitutions (Kaca et al., 1990; McCoy et al., 2001). Similarly, the genome of $P$. mirabilis has been shown to contain the eptC gene, which is involved in the modification of core LPSs with PEtN (Aquilini et al., 2014).

Polymyxin-susceptible mutants generated using transposon mutagenesis have been studied to gain insight into the mechanisms underlying polymyxin resistance in P. mirabilis. Some putative loci that may either directly or indirectly affect LPS modifications that have been identified to date include the sap operon (encoding a transport protein), the ATPase gene and a putative $O$-acetyltransferase gene (most likely involved in either the biosynthesis or transfer of aminoarabinose) (McCoy et al., 2001). The galU gene (involved in L-Ara4N biosynthesis) and a TCS termed $r p p A / r p p B$, which shares close similarity with the $p m r A / p m r B$ and $p h o P / p h o Q$ TCSs and is able to activate the $\operatorname{arnBCADTEF}$ operon, have been recently discovered to mediate polymyxin resistance in $P$. mirabilis (Wang et al., 2008; Jiang et al., 2010a,b). The inactivation of any of these genes in $P$. mirabilis results in a polymyxin-susceptible phenotype (Wang et al., 2008; Jiang et al., 2010a,b). For example, the transposomic inactivations of PpmrI (homologous to arnA of $S$. Typhimurium) and $r p p A$ (homologous to both the $p m r A$ and phoP of $S$. Typhimurium) genes of $P$. mirabilis resulted in 10240 and $>160$-fold susceptibility to polymyxin B, respectively, compared to the wild-type P. mirabilis strain (Wang et al., 2008; Jiang et al., 2010b).

Additionally, genomic analysis of intrinsically colistin-resistant Morganella morganii, which is closely related to Proteus spp., has revealed that this bacterium possesses an $\operatorname{arnBCADTEF}$ operon and $e p t B$ gene known to mediate the modifications of LPSs with L-Ara4N and PEtN, respectively (Olaitan et al., 2014a). The genome of M. morganii also contains most of the genes that have been implicated in intrinsic polymyxin resistance in P. mirabilis, such as the $r p p A / r p p B$ and $p h o P / p h o Q$ TCSs (Olaitan et al., 2014a). It is therefore possible that the Proteeae bacteria possess the same underlying mechanisms for polymyxin resistance.

\section{Serratia marcescens}

The inactivation of the $a r n B$ and $\operatorname{arn} C$ genes, which are part of the arnBCADTEF operon, has been shown to result in polymyxin sensitivity in Serratia marcescens mutants (Lin et al., 2014). The $\operatorname{arn} B$ and $\operatorname{arn} C$ mutants displayed a reduced susceptibility to polymyxin B with MIC from 2048 to $2 \mathrm{mg} / \mathrm{L}$ (a 1024-fold reduction) compared to the wild-type $S$. marcescens strain. This operon has been further reported to be regulated by phoP, which is the cognate response regulator of the $p h o P / p h o Q$ TCS (Lin et al., 
2014). Thus, these findings show that the modifications of the LPSs of $S$. marcescens as mediated by the arnBCADTEF operon are partly or wholly responsible for the intrinsic resistance of this bacterium to polymyxins.

The arnBCADTEF operon appears to be constitutively expressed in intrinsically resistant bacteria in contrast to nonintrinsically resistant bacteria. The principal question that remains to be answered is why this is true for these two sets of bacteria. An understanding of this mechanism will greatly aid in the elucidation of the evolution of polymyxin resistance in Gram-negative bacteria.

\section{Burkholderia species}

Generally, members of the genus Burkholderia comprising the Burkholderia cepacia complex (BCC) and many other
Burkholderia species are intrinsically resistant to polymyxins (Loutet and Valvano, 2011). L-Ara4N is a predominant part of both the lipid A and Kdo moieties of the LPSs of Burkholderia spp., including B. thailandensis and B. pseudomallei (Isshiki et al., 1998; Silipo et al., 2005; Novem et al., 2009). It is constitutively produced and is essential for these bacteria. A B. cenocepacia mutant lacking L-Ara4N has been observed to be highly susceptible to polymyxin B (Ortega et al., 2007). This unique composition together with the contributions of other genes, such as ispH (involved in the synthesis of isoprenoids), rpoE, norM (an efflux pump-encoding gene), and $h p n J$ (encodes hopanoid), play roles in the multifaceted mechanisms that result in high levels of polymyxin resistance in Burkholderia cepacia complex (Loutet and Valvano, 2011; Malott et al., 2012). An excellent review by Loutet et al. on the intrinsic resistance of Burkholderia cepacia

Table 2 | Strategies employed by Gram-negative bacteria for achieving resistance to polymyxins.

\begin{tabular}{|c|c|c|c|}
\hline Resistance mechanism & Genes involved & Bacteria & References \\
\hline $\begin{array}{l}\text { Modification of the lipid A or Kdo } \\
\text { with aminoarabinose }\end{array}$ & $\begin{array}{l}\text { arnBCADTEF operon } \\
\text { and pmrE }\end{array}$ & $\begin{array}{l}\text { Salmonella enterica, Klebsiella } \\
\text { pneumoniae, Escherichia coli, } \\
\text { Proteeae bacteria, Serratia } \\
\text { marcescens, Pseudomonas } \\
\text { aeruginosa and Burkholderia cepacia } \\
\text { complex }\end{array}$ & $\begin{array}{l}\text { Vaara et al., 1981; Boll et al., 1994; Nummila } \\
\text { et al., 1995; Helander et al., 1996; Rozalski } \\
\text { et al., 1997; Trent et al., 2001 b; Moskowitz } \\
\text { et al., 2004; Yan et al., 2007; Loutet and } \\
\text { Valvano, 2011; Lin et al., } 2014\end{array}$ \\
\hline
\end{tabular}

$\begin{array}{ll}\begin{array}{l}\text { Modification of the lipid A with } \quad \text { pmrC } \\ \text { phosphoethanolamine }\end{array} & \begin{array}{l}\text { S. enterica, K. pneumoniae, E. coli } \\ \text { and Acinetobacter baumannii }\end{array}\end{array}$
and Acinetobacter baumannii
Zhou et al., 2001; Lee et al., 2004; Kim et al., 2006; Arroyo et al., 2011; Beceiro et al., 2011; Jayol et al., 2014

Roland et al., 1993; Sun et al., 2009; Arroyo et al., 2011; Owusu-Anim and Kwon, 2012; Cannatelli et al., 2014b; Jayol et al., 2014 $\begin{array}{ll}\text { pmrA/pmrB and or } & \text { S. enterica, K. pneumoniae, } P \\ \text { phoP/phoQ } & \text { aeruginosa and A. baumannii }\end{array}$

$\begin{array}{ll}\text { pmrA/pmrB and or } & \text { S. enterica, K. pneumoniae, } P \\ \text { phoP/phoO } & \text { aeruginosa and A. baumannii }\end{array}$
Activation of LPS-modifying operon by mutations in TCSs

$$
\operatorname{mgr} B
$$

Inactivation of $p h o P / p h o Q$ negative feedback regulator

$$
\text { K. pneumoniae }
$$

eptB, phoP/phoQ E. coli and $m g r R$
IpxM

Increased acylation of lipid A

\begin{tabular}{|c|c|c|c|}
\hline $\begin{array}{l}\text { Modification of the Kdo with } \\
\text { phosphoethanolamine }\end{array}$ & $\begin{array}{l}\text { eptB, phoP/phoQ } \\
\text { and } \mathrm{mgrR}\end{array}$ & E. coli & $\begin{array}{l}\text { Reynolds et al., 2005; Moon and Gottes } \\
2009\end{array}$ \\
\hline $\begin{array}{l}\text { Increased acylation of lipid A } \\
\text { enhancing its modification with } \\
\text { aminoarabinose }\end{array}$ & IpxM & $\begin{array}{l}\text { S. enterica, K. pneumoniae and } \\
\text { E. coli }\end{array}$ & $\begin{array}{l}\text { Tran et al., 2005; Clements et al., 2007; } \\
\text { Murray et al., 2007; Velkov et al., 2013b }\end{array}$ \\
\hline
\end{tabular}
enhancing its modification with aminoarabinose
Cannatelli et al., 2013; López-Camacho et al., 2013; Gaibani et al., 2014; Olaitan et al., $2014 \mathrm{~b}$

Trapping of polymyxins by capsule

Efflux pump

myxins by capsule

\section{Loss of LPS}

$a c r A B$ and $k p n E F$

Campos et al., 2004; Llobet et al., 2008

K. pneumoniae

A. baumannii

A. baumannii

colR/cols, cprR/cprS P. aeruginosa and parR/parS
Padilla et al., 2010; Srinivasan and

Rajamohan, 2013

Moffatt et al., 2010, 2011

Pelletier et al., 2013

Glycosylation of lipid A with

hexosamine

Acquired/adaptive resistance to polymyxins through LPS

modification with aminoarabinose

\begin{tabular}{lll}
\hline $\begin{array}{l}\text { Overexpression of outer membrane } \\
\text { protein OprH }\end{array}$ & oprH & P. aeruginosang et al., 1992
\end{tabular}

Fernández et al., 2010; Muller et al., 2011;

Fernandez et al., 2012; Gutu et al., 2013

protein $\mathrm{OprH}$ 
complex to polymyxins explains the roles of some of these genes (Loutet and Valvano, 2011).

\section{CONCLUSIONS}

Resistance to polymyxin antibiotics depends on an intricate mechanism that involves several genes participating in bacterial cell membrane remodeling. However, most of the strategies employed by bacteria target the cell surface, which compromises its integrity or results in its modification, as summarized in Table 2. Although a large amount of work aimed at elucidating the mechanisms underlying polymyxin resistance has been performed, a great deal of information remains to be discovered because resistant strains exist for which the mechanisms underlying their resistance are unknown. Several other candidate genes have also been described, but their contributions to polymyxin resistance have not been fully examined. Such findings are of paramount importance given the current significance of polymyxins in clinical practice and the increased bacterial resistance to these drugs.

\section{AUTHOR CONTRIBUTIONS}

Conceived and designed the study: Jean-Marc Rolain and Serge Morand; analyzed the data: Abiola O. Olaitan and Jean-Marc Rolain; wrote the paper: Abiola O. Olaitan and Jean-Marc Rolain.

\section{ACKNOWLEDGMENTS}

The authors wish to acknowledge the Infectiopole Sud Foundation for funding Abiola O. Olaitan's Ph.D. thesis. We also thank American Journal Experts for their editing services.

\section{REFERENCES}

Abraham, N., and Kwon, D. H. (2009). A single amino acid substitution in PmrB is associated with polymyxin B resistance in clinical isolate of Pseudomonas aeruginosa. FEMS Microbiol. Lett. 298, 249-254. doi: 10.1111/j.1574-6968.2009.01720.x

Adams, M. D., Nickel, G. C., Bajaksouzian, S., Lavender, H., Murthy, A. R., Jacobs, M. R., et al. (2009). Resistance to colistin in Acinetobacter baumannii associated with mutations in the PmrAB two-component system. Antimicrob. Agents Chemother. 53, 3628-3634. doi: 10.1128/AAC.00284-09

Agerso, Y., Torpdahl, M., Zachariasen, C., Seyfarth, A., Hammerum, A. M., and Nielsen, E. M. (2012). Tentative colistin epidemiological cut-off value for Salmonella spp. Foodborne Pathog. Dis. 9, 367-369. doi: 10.1089/fpd.2011.1015

Aguirre, A., Lejona, S., Vescovi, E. G., and Soncini, F. C. (2000). Phosphorylated PmrA interacts with the promoter region of ugd in Salmonella enterica serovar typhimurium. J. Bacteriol. 182, 3874-3876. doi: 10.1128/JB.182.13.38743876.2000

Aquilini, E., Merino, S., Knirel, Y. A., Regue, M., and Tomas, J. M. (2014). Functional identification of Proteus mirabilis eptC gene encoding a core lipopolysaccharide phosphoethanolamine transferase. Int. J. Mol. Sci. 15, 6689-6702. doi: 10.3390/ijms 15046689

Arduino, S. M., Quiroga, M. P., Ramirez, M. S., Merkier, A. K., Errecalde, L., Di, M. A., et al. (2012). Transposons and integrons in colistin-resistant clones of Klebsiella pneumoniae and Acinetobacter baumannii with epidemic or sporadic behaviour. J. Med. Microbiol. 61, 1417-1420. doi: 10.1099/jmm.0.038968-0

Arroyo, L. A., Herrera, C. M., Fernandez, L., Hankins, J. V., Trent, M. S., and Hancock, R. E. (2011). The pmrCAB operon mediates polymyxin resistance in Acinetobacter baumannii ATCC 17978 and clinical isolates through phosphoethanolamine modification of lipid A. Antimicrob. Agents Chemother. 55, 3743-3751. doi: 10.1128/AAC.00256-11

Barchiesi, J., Espariz, M., Checa, S. K., and Soncini, F. C. (2009). Downregulation of RpoN-controlled genes protects Salmonella cells from killing by the cationic antimicrobial peptide polymyxin B. FEMS Microbiol. Lett. 291, 73-79. doi: 10.1111/j.1574-6968.2008.01437.x
Barin, J., Martins, A. F., Heineck, B. L., Barth, A. L., and Zavascki, A. P. (2013). Hetero- and adaptive resistance to polymyxin B in OXA-23-producing carbapenem-resistant Acinetobacter baumannii isolates. Ann. Clin. Microbiol. Antimicrob. 12, 15. doi: 10.1186/1476-0711-12-15

Barrow, K., and Kwon, D. H. (2009). Alterations in two-component regulatory systems of phoPQ and pmrAB are associated with polymyxin B resistance in clinical isolates of Pseudomonas aeruginosa. Antimicrob. Agents Chemother. 53, 5150-5154. doi: 10.1128/AAC.00893-09

Basu, S., Radziejewska-Lebrecht, J., and Mayer, H. (1986). Lipopolysaccharide of Providencia rettgeri. Chemical studies and taxonomical implications. Arch. Microbiol. 144, 213-218. doi: 10.1007/BF00410949

Beceiro, A., Llobet, E., Aranda, J., Bengoechea, J. A., Doumith, M., Hornsey, M., et al. (2011). Phosphoethanolamine modification of lipid A in colistinresistant variants of Acinetobacter baumannii mediated by the pmrAB twocomponent regulatory system. Antimicrob. Agents Chemother. 55, 3370-3379. doi: 10.1128/AAC.00079-11

Beceiro, A., Moreno, A., Fernandez, N., Vallejo, J. A., Aranda, J., Adler, B., et al. (2014). Biological cost of different mechanisms of colistin resistance and their impact on virulence in Acinetobacter baumannii. Antimicrob. Agents Chemother. 58, 518-526. doi: 10.1128/AAC.01597-13

Biswas, S., Brunel, J. M., Dubus, J. C., Reynaud-Gaubert, M., and Rolain, J. M. (2012). Colistin: an update on the antibiotic of the 21st century. Expert Rev. Anti. Infect. Ther. 10, 917-934. doi: 10.1586/eri.12.78

Boll, M., Radziejewska-Lebrecht, J., Warth, C., Krajewska-Pietrasik, D., and Mayer, H. (1994). 4-Amino-4-deoxy-L-arabinose in LPS of enterobacterial R-mutants and its possible role for their polymyxin reactivity. FEMS Immunol. Med. Microbiol. 8, 329-341.

Cai, Y., Chai, D., Wang, R., Liang, B., and Bai, N. (2012). Colistin resistance of Acinetobacter baumannii: clinical reports, mechanisms and antimicrobial strategies. J. Antimicrob. Chemother. 67, 1607-1615. doi: 10.1093/jac/dks084

Campos, M. A., Vargas, M. A., Regueiro, V., Llompart, C. M., Alberti, S., and Bengoechea, J. A. (2004). Capsule polysaccharide mediates bacterial resistance to antimicrobial peptides. Infect. Immun. 72, 7107-7114. doi: 10.1128/IAI.72.12.7107-7114.2004

Cannatelli, A., D'Andrea, M. M., Giani, T., Di, P. V., Arena, F., Ambretti, S., et al. (2013). In vivo emergence of colistin resistance in Klebsiella pneumoniae producing KPC-type carbapenemases mediated by insertional inactivation of the PhoQ/PhoP mgrB regulator. Antimicrob. Agents Chemother. 57, 5521-5526. doi: 10.1128/AAC.01480-13

Cannatelli, A., Giani, T., D’Andrea, M. M., Di Pilato, V., Arena, F., Conte, V., et al. (2014a). MgrB inactivation is a common mechanism of colistin resistance in KPC carbapenemase-producing Klebsiella pneumoniae of clinical origin. Antimicrob. Agents Chemother. 58, 5696-5703. doi: 10.1128/AAC.03110-14

Cannatelli, A., Di Pilato, V., Giani, T., Arena, F., Ambretti, S., Gaibani, P., et al. (2014b). In vivo evolution to colistin resistance by PmrB sensor kinase mutation in KPC carbapenemase-producing Klebsiella pneumoniae associated with lowdosage colistin treatment. Antimicrob. Agents Chemother. 58, 4399-4403. doi: 10.1128/AAC.02555-14

Cheng, H. Y., Chen, Y. F., and Peng, H. L. (2010). Molecular characterization of the PhoPQ-PmrD-PmrAB mediated pathway regulating polymyxin B resistance in Klebsiella pneumoniae CG43. J. Biomed. Sci. 17:60. doi: 10.1186/14230127-17-60

Choi, M. J., and Ko, K. S. (2014). Mutant prevention concentrations of colistin for Acinetobacter baumannii, Pseudomonas aeruginosa and Klebsiella pneumoniae clinical isolates. J. Antimicrob. Chemother. 69, 275-277. doi: 10.1093/jac/ dkt315

Clements, A., Tull, D., Jenney, A. W., Farn, J. L., Kim, S. H., Bishop, R. E., et al. (2007). Secondary acylation of Klebsiella pneumoniae lipopolysaccharide contributes to sensitivity to antibacterial peptides. J. Biol. Chem. 282, 15569-15577. doi: 10.1074/jbc.M701454200

Coornaert, A., Lu, A., Mandin, P., Springer, M., Gottesman, S., and Guillier, M. (2010). MicA sRNA links the PhoP regulon to cell envelope stress. Mol. Microbiol. 76, 467-479. doi: 10.1111/j.1365-2958.2010.07115.x

de, J. A., Thomas, V., Simjee, S., Godinho, K., Schiessl, B., Klein, U., et al. (2012). Pan-European monitoring of susceptibility to human-use antimicrobial agents in enteric bacteria isolated from healthy food-producing animals. J. Antimicrob. Chemother. 67, 638-651. doi: 10.1093/jac/dkr539

Diene, S. M., Merhej, V., Henry, M., El, F. A., Roux, V., Robert, C., et al. (2013). The rhizome of the multidrug-resistant Enterobacter aerogenes genome reveals how 
new "killer bugs" are created because of a sympatric lifestyle. Mol. Biol. Evol. 30, 369-383. doi: 10.1093/molbev/mss236

EMEA. (2002). Colistin: Summary report (1), EMEA/MRL/016/95-FINAL. London: The European Agency for the Evaluation of Medicinal Products, Committee for Veterinary Medicinal Products.

Ernst, R. K., Guina, T., and Miller, S. I. (2001). Salmonella Typhimurium outer membrane remodeling: role in resistance to host innate immunity. Microbes Infect. 3, 1327-1334.

Falagas, M. E., and Michalopoulos, A. (2006). Polymyxins: old antibiotics are back. Lancet 367, 633-634. doi: 10.1016/S0140-6736(06)68241-X

Falagas, M. E., Rafailidis, P. I., and Matthaiou, D. K. (2010). Resistance to polymyxins: Mechanisms, frequency and treatment options. Drug Resist. Updat. 13, 132-138. doi: 10.1016/j.drup.2010.05.002

Fernandez, L., Alvarez-Ortega, C., Wiegand, I., Olivares, J., Kocincova, D., Lam, J. S., et al. (2013). Characterization of the polymyxin B resistome of Pseudomonas aeruginosa. Antimicrob. Agents Chemother. 57, 110-119. doi: 10.1128/AAC.01583-12

Fernández, L., Gooderham, W. J., Bains, M., McPhee, J. B., Wiegand, I., and Hancock, R. E. W. (2010). Adaptive resistance to the "last hope" antibiotics polymyxin B and colistin in Pseudomonas aeruginosa is mediated by the novel two-component regulatory system ParR-ParS. Antimicrob. Agents Chemother. 54, 3372-3382. doi: 10.1128/AAC.00242-10

Fernandez, L., Jenssen, H., Bains, M., Wiegand, I., Gooderham, W. J., and Hancock, R. E. (2012). The two-component system CprRS senses cationic peptides and triggers adaptive resistance in Pseudomonas aeruginosa independently of ParRS. Antimicrob. Agents Chemother. 56, 6212-6222. doi: 10.1128/AAC.01530-12

Fernandez-Reyes, M., Rodriguez-Falcon, M., Chiva, C., Pachon, J., Andreu, D., and Rivas, L. (2009). The cost of resistance to colistin in Acinetobacter baumannii: a proteomic perspective. Proteomics 9, 1632-1645. doi: 10.1002/pmic.200800434

Gaibani, P., Lombardo, D., Lewis, R. E., Mercuri, M., Bonora, S., Landini, M. P., et al. (2014). In vitro activity and post-antibiotic effects of colistin in combination with other antimicrobials against colistin-resistant KPC-producing Klebsiella pneumoniae bloodstream isolates. J. Antimicrob. Chemother. 69, 1856-1865. doi: 10.1093/jac/dku065

Guckes, K. R., Kostakioti, M., Breland, E. J., Gu, A. P., Shaffer, C. L., Martinez, C. R. III, et al. (2013). Strong cross-system interactions drive the activation of the QseB response regulator in the absence of its cognate sensor. Proc. Natl. Acad. Sci. U.S.A. 110, 16592-16597. doi: 10.1073/pnas. 1315320110

Gunn, J. S. (2001). Bacterial modification of LPS and resistance to antimicrobial peptides. J. Endotoxin. Res. 7, 57-62. doi: 10.1177/096805190100700 11001

Gunn, J. S., Lim, K. B., Krueger, J., Kim, K., Guo, L., Hackett, M., et al. (1998). PmrA-PmrB-regulated genes necessary for 4-aminoarabinose lipid A modification and polymyxin resistance. Mol. Microbiol. 27, 1171-1182. doi: 10.1046/j.1365-2958.1998.00757.x

Gunn, J. S., and Miller, S. I. (1996). PhoP-PhoQ activates transcription of pmrAB, encoding a two-component regulatory system involved in Salmonella typhimurium antimicrobial peptide resistance. J. Bacteriol. 178, 6857-6864.

Gunn, J. S., Ryan, S. S., Van Velkinburgh, J. C., Ernst, R. K., and Miller, S. I. (2000). Genetic and functional analysis of a PmrA-PmrB-regulated locus necessary for lipopolysaccharide modification, antimicrobial peptide resistance, and oral virulence of Salmonella enterica serovar typhimurium. Infect. Immun. 68 , 6139-6146. doi: 10.1128/IAI.68.11.6139-6146.2000

Guo, L., Lim, K. B., Gunn, J. S., Bainbridge, B., Darveau, R. P., Hackett, M., et al. (1997). Regulation of lipid A modifications by Salmonella typhimurium virulence genes phoP-phoQ. Science 276, 250-253. doi: 10.1126/science. 276.5310 .250

Gutu, A. D., Sgambati, N., Strasbourger, P., Brannon, M. K., Jacobs, M. A., Haugen, E., et al. (2013). Polymyxin resistance of Pseudomonas aeruginosa phoQ mutants is dependent on additional two-component regulatory systems. Antimicrob. Agents Chemother. 57, 2204-2215. doi: 10.1128/AAC.02353-12

Hayakawa, K., Marchaim, D., Divine, G. W., Pogue, J. M., Kumar, S., Lephart, P., et al. (2012). Growing prevalence of Providencia stuartii associated with the increased usage of colistin at a tertiary health care center. Int. J. Infect. Dis. 16, e646-e648. doi: 10.1016/j.iiid.2012.05.1029

Helander, I. M., Kato, Y., Kilpelainen, I., Kostiainen, R., Lindner, B., Nummila, K., et al. (1996). Characterization of lipopolysaccharides of polymyxin-resistant and polymyxin-sensitive Klebsiella pneumoniae O3. Eur. J. Biochem. 237, 272-278. doi: 10.1111/j.1432-1033.1996.0272n.x
Helander, I. M., Kilpelainen, I., and Vaara, M. (1994). Increased substitution of phosphate groups in lipopolysaccharides and lipid A of the polymyxin-resistant pmrA mutants of Salmonella typhimurium: a 31P-NMR study. Mol. Microbiol. 11, 481-487. doi: 10.1111/j.1365-2958.1994.tb00329.x

Herrera, C. M., Hankins, J. V., and Trent, M. S. (2010). Activation of PmrA inhibits LpxT-dependent phosphorylation of lipid A promoting resistance to antimicrobial peptides. Mol. Microbiol. 76, 1444-1460. doi: 10.1111/j.13652958.2010.07150.x

Hood, M. I., Becker, K. W., Roux, C. M., Dunman, P. M., and Skaar, E. P. (2013). genetic determinants of intrinsic colistin tolerance in Acinetobacter baumannii. Infect. Immun. 81, 542-551. doi: 10.1128/IAI.00704-12

Hraiech, S., Roch, A., Lepidi, H., Atieh, T., Audoly, G., Rolain, J. M., et al. (2013). Impaired virulence and fitness of a colistin-resistant clinical isolate of Acinetobacter baumannii in a rat model of pneumonia. Antimicrob. Agents Chemother. 57, 5120-5121. doi: 10.1128/AAC.00700-13

Isshiki, Y., Kawahara, K., and Zahringer, U. (1998). Isolation and characterisation of disodium (4-amino-4-deoxy-beta-L- arabinopyranosyl)-(1-->8)-(Dglycero-alpha-D-talo-oct-2-ulopyranosylona te)- (2-->4)-(methyl 3deoxy-D-manno-oct-2-ulopyranosid)onate from the lipopolysaccharide of Burkholderia cepacia. Carbohydr. Res. 313, 21-27. doi: 10.1016/S0008-6215(98) 00179-7

Jayol, A., Poirel, L., Brink, A., Villegas, M.-V., Yilmaz, M., and Nordmann, P. (2014). Resistance to colistin associated to a single amino acid change in protein PmrB among Klebsiella pneumoniae isolates of worldwide origin. Antimicrob. Agents Chemother. 58, 4762-4766. doi: 10.1128/AAC.00084-14

Jiang, S. S., Lin, T. Y., Wang, W. B., Liu, M. C., Hsueh, P. R., and Liaw, S. J. (2010a), Characterization of UDP-glucose dehydrogenase and UDP-glucose pyrophosphorylase mutants of Proteus mirabilis: defectiveness in polymyxin B resistance, swarming, and virulence. Antimicrob. Agents Chemother. 54, 2000-2009. doi: 10.1128/AAC.01384-09

Jiang, S. S., Liu, M. C., Teng, L. J., Wang, W. B., Hsueh, P. R., and Liaw, S. J. (2010b). Proteus mirabilis pmrI, an RppA-regulated gene necessary for polymyxin B resistance, biofilm formation, and urothelial cell invasion. Antimicrob. Agents Chemother. 54, 1564-1571. doi: 10.1128/AAC.01219-09

Johansen, H. K., Moskowitz, S. M., Ciofu, O., Pressler, T., and Hoiby, N. (2008). Spread of colistin resistant non-mucoid Pseudomonas aeruginosa among chronically infected Danish cystic fibrosis patients. J. Cyst. Fibros. 7, 391-397. doi: 10.1016/j.jcf.2008.02.003

Jones, J. W., Shaffer, S. A., Ernst, R. K., Goodlett, D. R., and Turecek, F. (2008). Determination of pyrophosphorylated forms of lipid A in Gram-negative bacteria using a multivaried mass spectrometric approach. Proc. Natl. Acad. Sci. U.S.A. 105, 12742-12747. doi: 10.1073/pnas.0800445105

Kaca, W., Radziejewska-Lebrecht, J., and Bhat, U. R. (1990). Effect of polymyxins on the lipopolysaccharide-defective mutants of Proteus mirabilis. Microbios $61,23-32$.

Kato, A., Chen, H. D., Latifi, T., and Groisman, E. A. (2012). Reciprocal control between a bacterium's regulatory system and the modification status of its lipopolysaccharide. Mol. Cell 47, 897-908. doi: 10.1016/j.molcel.2012. 07.017

Kato, A., Latifi, T., and Groisman, E. A. (2003). Closing the loop: the PmrA/PmrB two-component system negatively controls expression of its posttranscriptional activator PmrD. Proc. Natl. Acad. Sci. U.S.A. 100, 4706-4711. doi: $10.1073 /$ pnas. 0836837100

Kawasaki, K., China, K., and Nishijima, M. (2007). Release of the lipopolysaccharide deacylase PagL from latency compensates for a lack of lipopolysaccharide aminoarabinose modification-dependent resistance to the antimicrobial peptide polymyxin B in Salmonella enterica. J. Bacteriol. 189, 4911-4919. doi: 10.1128/JB.00451-07

Kawasaki, K., Ernst, R. K., and Miller, S. I. (2005). Inhibition of Salmonella enterica serovar Typhimurium lipopolysaccharide deacylation by aminoarabinose membrane modification. J. Bacteriol. 187, 2448-2457. doi: 10.1128/JB.187.7.24482457.2005

Kazmierczak, M. J., Wiedmann, M., and Boor, K. J. (2005). Alternative sigma factors and their roles in bacterial virulence. Microbiol. Mol. Biol. Rev. 69, 527-543. doi: 10.1128/MMBR.69.4.527-543.2005

Kempf, I., Fleury, M. A., Drider, D., Bruneau, M., Sanders, P., Chauvin, C., et al. (2013). What do we know about resistance to colistin in Enterobacteriaceae in avian and pig production in Europe? Int. J. Antimicrob. Agents 42, 379-383. doi: 10.1016/j.ijantimicag.2013.06.012 
Kim, S. H., Jia, W., Parreira, V. R., Bishop, R. E., and Gyles, C. L. (2006). Phosphoethanolamine substitution in the lipid A of Escherichia coli O157: H7 and its association with PmrC. Microbiology 152, 657-666. doi: 10.1099/mic.0. 28692-0

Kim, S. Y., Choi, H. J., and Ko, K. S. (2014a). Differential expression of twocomponent systems, $\mathrm{pmrAB}$ and phoPQ, with different growth phases of Klebsiella pneumoniae in the presence or absence of colistin. Curr. Microbiol. 69, 37-41. doi: 10.1007/s00284-014-0549-0

Kim, Y., Bae, I. K., Lee, H., Jeong, S. H., Yong, D., and Lee, K. (2014b). In vivo emergence of colistin resistance in Acinetobacter baumannii clinical isolates of sequence type 357 during colistin treatment. Diagn. Microbiol. Infect. Dis. 79, 362-366. doi: 10.1016/j.diagmicrobio.2014.03.027

Kox, L. F., Wosten, M. M., and Groisman, E. A. (2000). A small protein that mediates the activation of a two-component system by another two-component system. EMBO J. 19, 1861-1872. doi: 10.1093/emboj/19.8.1861

Lacour, S., Bechet, E., Cozzone, A. J., Mijakovic, I., and Grangeasse, C. (2008). Tyrosine phosphorylation of the UDP-glucose dehydrogenase of Escherichia coli is at the crossroads of colanic acid synthesis and polymyxin resistance. PLoS ONE 3:e3053. doi: 10.1371/journal.pone.0003053

Lacour, S., Doublet, P., Obadia, B., Cozzone, A. J., and Grangeasse, C. (2006). A novel role for protein-tyrosine kinase Etk from Escherichia coli K-12 related to polymyxin resistance. Res. Microbiol. 157, 637-641. doi: 10.1016/j.resmic.2006.01.003

Lean, S. S., Suhaili, Z., Ismail, S., Rahman, N. I., Othman, N., Abdullah, F. H., et al. (2014). Prevalence and genetic characterization of carbapenemand polymyxin-resistant Acinetobacter baumannii Isolated from a tertiary hospital in Terengganu, Malaysia. ISRN. Microbiol. 2014, 953417. doi: $10.1155 / 2014 / 953417$

Lee, H., Hsu, F. F., Turk, J., and Groisman, E. A. (2004). The PmrA-regulated pmrC gene mediates phosphoethanolamine modification of lipid $\mathrm{A}$ and polymyxin resistance in Salmonella enterica. J. Bacteriol. 186, 4124-4133. doi: 10.1128/JB.186.13.4124-4133.2004

Lee, J.-Y., Chung, E. S., Na, I. Y., Kim, H., Shin, D., and Ko, K. S. (2014a). Development of colistin resistance in pmrA-, phoP-, parR- and cprR-inactivated mutants of Pseudomonas aeruginosa. J. Antimicrob. Chemother. 69, 2966-2971. doi: $10.1093 / \mathrm{jac} / \mathrm{dku} 238$

Lee, J. Y., and Ko, K. S. (2014). Mutations and expression of PmrAB and PhoPQ related with colistin resistance in Pseudomonas aeruginosa clinical isolates. Diagn. Microbiol. Infect. Dis. 78, 271-276. doi: 10.1016/j.diagmicrobio.2013. 11.027

Lee, J. Y., Na, I. Y., Park, Y. K., and Ko, K. S. (2014b). Genomic variations between colistin-susceptible and -resistant Pseudomonas aeruginosa clinical isolates and their effects on colistin resistance. J. Antimicrob. Chemother. 69, 1248-1256. doi: $10.1093 / \mathrm{jac} / \mathrm{dkt} 531$

Lesho, E., Yoon, E. J., McGann, P., Snesrud, E., Kwak, Y., Milillo, M., et al. (2013). Emergence of colistin-resistance in extremely drug-resistant Acinetobacter baumannii containing a novel pmrCAB operon during colistin therapy of wound infections. J. Infect. Dis. 208, 1142-1151. doi: 10.1093/infdis/jit293

Li, J., Rayner, C. R., Nation, R. L., Owen, R. J., Spelman, D., Tan, K. E., et al. (2006). Heteroresistance to colistin in multidrug-resistant Acinetobacter baumannii. Antimicrob. Agents Chemother. 50, 2946-2950. doi: 10.1128/AAC. 00103-06

Lim, L. M., Ly, N., Anderson, D., Yang, J. C., Macander, L., Jarkowski, A. III, et al. (2010). Resurgence of colistin: a review of resistance, toxicity, pharmacodynamics, and dosing. Pharmacotherapy 30, 1279-1291. doi: 10.1592/phco.30.12.1279

Lin, Q. Y., Tsai, Y.-L., Liu, M.-C., Lin, W.-C., Hsueh, P.-R., and Liaw, S.-J. (2014). Serratia marcescens arn, a PhoP-regulated locus necessary for polymyxin B resistance. Antimicrob. Agents Chemother. 58, 5181-5190. doi: 10.1128/AAC. 00013-14

Lippa, A. M., and Goulian, M. (2009). Feedback inhibition in the PhoQ/PhoP signaling system by a membrane peptide. PLoS. Genet. 5:e1000788. doi: 10.1371/journal.pgen.1000788

Lippa, A. M., and Goulian, M. (2012). Perturbation of the oxidizing environment of the periplasm stimulates the $\mathrm{PhoQ} / \mathrm{PhoP}$ system in Escherichia coli. J. Bacteriol. 194, 1457-1463. doi: 10.1128/JB.06055-11

Llobet, E., Campos, M. A., Gimenez, P., Moranta, D., and Bengoechea, J. A. (2011). Analysis of the networks controlling the antimicrobial-peptidedependent induction of Klebsiella pneumoniae virulence factors. Infect. Immun. 79, 3718-3732. doi: 10.1128/IAI.05226-11
Llobet, E., Tomas, J. M., and Bengoechea, J. A. (2008). Capsule polysaccharide is a bacterial decoy for antimicrobial peptides. Microbiology 154, 3877-3886. doi: 10.1099/mic.0.2008/022301-0

López-Camacho, E., Gómez-Gil, R., Tobes, R., Manrique, M., Lorenzo, M., Galván, B., et al. (2013). Genomic analysis of the emergence and evolution of multidrug resistance during a Klebsiella pneumoniae outbreak including carbapenem and colistin resistance. J. Antimicrob. Chemother. 69, 632-636. doi: 10.1093/jac/ dkt419

Lopez-Rojas, R., Dominguez-Herrera, J., McConnell, M. J., Docobo-Perez, F., Smani, Y., Fernandez-Reyes, M., et al. (2011). Impaired virulence and in vivo fitness of colistin-resistant Acinetobacter baumannii. J. Infect. Dis. 203, 545-548. doi: 10.1093/infdis/jiq086

Loutet, S. A., and Valvano, M. A. (2011). Extreme antimicrobial peptide and polymyxin B resistance in the genus Burkholderia. Front. Cell Infect. Microbiol. 1:6. doi: $10.3389 /$ fcimb. 2011.00006

Macfarlane, E. L., Kwasnicka, A., and Hancock, R. E. (2000). Role of Pseudomonas aeruginosa PhoP-phoQ in resistance to antimicrobial cationic peptides and aminoglycosides. Microbiology 146(Pt 10), 2543-2554.

Macfarlane, E. L., Kwasnicka, A., Ochs, M. M., and Hancock, R. E. (1999). PhoP-PhoQ homologues in Pseudomonas aeruginosa regulate expression of the outer-membrane protein OprH and polymyxin B resistance. Mol. Microbiol. 34, 305-316. doi: 10.1046/j.1365-2958.1999.01600.x

Malott, R. J., Steen-Kinnaird, B. R., Lee, T. D., and Speert, D. P. (2012). Identification of hopanoid biosynthesis genes involved in polymyxin resistance in Burkholderia multivorans. Antimicrob. Agents Chemother. 56, 464-471. doi: 10.1128/AAC.00602-11

Mammina, C., Bonura, C., Di, B. F., Aleo, A., Fasciana, T., Sodano, C., et al. (2012). Ongoing spread of colistin-resistant Klebsiella pneumoniae in different wards of an acute general hospital, Italy, June to December 2011. Euro. Surveill 17.

McCoy, A. J., Liu, H., Falla, T. J., and Gunn, J. S. (2001). Identification of Proteus mirabilis mutants with increased sensitivity to antimicrobial peptides. Antimicrob. Agents Chemother. 45, 2030-2037. doi: 10.1128/AAC.45.7.20302037.2001

McPhee, J. B., Lewenza, S., and Hancock, R. E. (2003). Cationic antimicrobial peptides activate a two-component regulatory system, $\mathrm{PmrA}-\mathrm{PmrB}$, that regulates resistance to polymyxin B and cationic antimicrobial peptides in Pseudomonas aeruginosa. Mol. Microbiol. 50, 205-217. doi: 10.1046/j.1365-2958.2003.03673.x

Merkier, A. K., Rodriguez, M. C., Togneri, A., Brengi, S., Osuna, C., Pichel, M., et al. (2013). Outbreak of a cluster with epidemic behavior due to Serratia marcescens after colistin administration in a hospital setting. J. Clin. Microbiol. 51, 2295-2302. doi: 10.1128/JCM.03280-12

Miller, A. K., Brannon, M. K., Stevens, L., Johansen, H. K., Selgrade, S. E., Miller, S. I., et al. (2011). PhoQ mutations promote lipid A modification and polymyxin resistance of Pseudomonas aeruginosa found in colistin-treated cystic fibrosis patients. Antimicrob. Agents Chemother. 55, 5761-5769. doi: 10.1128/AAC.05391-11

Mitrophanov, A. Y., Jewett, M. W., Hadley, T. J., and Groisman, E. A. (2008). Evolution and dynamics of regulatory architectures controlling polymyxin B resistance in enteric bacteria. PLoS. Genet. 4:e1000233. doi: 10.1371/journal.pgen. 1000233

Moffatt, J. H., Harper, M., Adler, B., Nation, R. L., Li, J., and Boyce, J. D. (2011). Insertion sequence ISAball is involved in colistin resistance and loss of lipopolysaccharide in Acinetobacter baumannii. Antimicrob. Agents Chemother. 55, 3022-3024. doi: 10.1128/AAC.01732-10

Moffatt, J. H., Harper, M., Harrison, P., Hale, J. D., Vinogradov, E., Seemann, T., et al. (2010). Colistin resistance in Acinetobacter baumannii is mediated by complete loss of lipopolysaccharide production. Antimicrob. Agents Chemother. 54, 4971-4977. doi: 10.1128/AAC.00834-10

Moon, K., and Gottesman, S. (2009). A PhoQ/P-regulated small RNA regulates sensitivity of Escherichia coli to antimicrobial peptides. Mol. Microbiol. 74, 1314-1330. doi: 10.1111/j.1365-2958.2009.06944.x

Morales, A. S., Fragoso de, A. J., de Moura Gomes, V. T., Reis Costa, A. T., dos Prazeres, R. D., Porfida Ferreira, T. S., et al. (2012). Colistin resistance in Escherichia coli and Salmonella enterica strains isolated from swine in Brazil. ScientificWorldJournal 2012:109795. doi: 10.1100/2012/109795

Moskowitz, S. M., Brannon, M. K., Dasgupta, N., Pier, M., Sgambati, N., Miller, A. K., et al. (2012). PmrB mutations promote polymyxin resistance of Pseudomonas aeruginosa isolated from colistin-treated cystic fibrosis patients. Antimicrob. Agents Chemother. 56, 1019-1030. doi: 10.1128/AAC.05829-11 
Moskowitz, S. M., Ernst, R. K., and Miller, S. I. (2004). PmrAB, a two-component regulatory system of Pseudomonas aeruginosa that modulates resistance to cationic antimicrobial peptides and addition of aminoarabinose to lipid A. J. Bacteriol. 186, 575-579. doi: 10.1128/JB.186.2.575-579.2004

Muller, C., Plesiat, P., and Jeannot, K. (2011). A two-component regulatory system interconnects resistance to polymyxins, aminoglycosides, fluoroquinolones, and beta-lactams in Pseudomonas aeruginosa. Antimicrob. Agents Chemother. 55, 1211-1221. doi: 10.1128/AAC.01252-10

Murray, S. R., Ernst, R. K., Bermudes, D., Miller, S. I., and Low, K. B. (2007). pmrA(Con) confers pmrHFIJKL-dependent EGTA and polymyxin resistance on msbB Salmonella by decorating lipid A with phosphoethanolamine. J. Bacteriol. 189, 5161-5169. doi: 10.1128/JB.01969-06

Muyembe, T., Vandepitte, J., and Desmyter, J. (1973). Natural colistin resistance in Edwardsiella tarda. Antimicrob. Agents Chemother. 4, 521-524. doi: 10.1128/AAC.4.5.521

Nation, R. L., and Li, J. (2009). Colistin in the 21st century. Curr. Opin. Infect. Dis. 22, 535-543. doi: 10.1097/QCO.0b013e328332e672

Nikaido, H. (2003). Molecular basis of bacterial outer membrane permeability revisited. Microbiol. Mol. Biol. Rev. 67, 593-656. doi: 10.1128/MMBR.67.4.593656.2003

Novem, V., Shui, G., Wang, D., Bendt, A. K., Sim, S. H., Liu, Y., et al. (2009). Structural and biological diversity of lipopolysaccharides from Burkholderia pseudomallei and Burkholderia thailandensis. Clin. Vaccine Immunol. 16, 1420-1428. doi: 10.1128/CVI.00472-08

Nummila, K., Kilpelainen, I., Zahringer, U., Vaara, M., and Helander, I. M. (1995). Lipopolysaccharides of polymyxin B-resistant mutants of Escherichia coli are extensively substituted by 2 -aminoethyl pyrophosphate and contain aminoarabinose in lipid A. Mol. Microbiol. 16, 271-278. doi: 10.1111/j.13652958.1995.tb02299.x

Olaitan, A. O., Diene, S. M., Gupta, S. K., Adler, A., Assous, M. V., and Rolain, J.-M. (2014a). Genome analysis of NDM-1 producing Morganella morganii clinical isolate. Expert Rev. Anti. Infect. Ther. 12, 1297-1305. doi: 10.1586/14787210. 2014.944504

Olaitan, A. O., Diene, S. M., Kempf, M., Berrazeg, M., Bakour, S., Gupta, S. K., et al. (2014b). Worldwide emergence of colistin resistance in Klebsiella pneumoniae from healthy humans and patients in Lao PDR, Thailand, Israel, Nigeria and France owing to inactivation of the PhoP/PhoQ regulator mgrB: an epidemiological and molecular study. Int. J. Antimicrob. Agents. doi: 10.1016/j. ijantimicag.2014.07.020. [Epub ahead of print].

Ortega, X. P., Cardona, S. T., Brown, A. R., Loutet, S. A., Flannagan, R. S., Campopiano, D. J., et al. (2007). A putative gene cluster for aminoarabinose biosynthesis is essential for Burkholderia cenocepacia viability. J. Bacteriol. 189, 3639-3644. doi: 10.1128/JB.00153-07

Owusu-Anim, D., and Kwon, D. H. (2012). Differential role of two-component regulatory systems (phoPQ and pmrAB) in polymyxin B susceptibility of Pseudomonas aeruginosa. Adv. Microbiol. 2. doi: 10.4236/aim.2012.21005

Padilla, E., Llobet, E., Domenech-Sanchez, A., Martinez-Martinez, L., Bengoechea, J. A., and Alberti, S. (2010). Klebsiella pneumoniae AcrAB efflux pump contributes to antimicrobial resistance and virulence. Antimicrob. Agents Chemother. 54, 177-183. doi: 10.1128/AAC.00715-09

Park, Y. K., Choi, J. Y., Shin, D., and Ko, K. S. (2011). Correlation between overexpression and amino acid substitution of the PmrAB locus and colistin resistance in Acinetobacter baumannii. Int. J. Antimicrob. Agents 37, 525-530. doi: 10.1016/j.ijantimicag.2011.02.008

Pelletier, M. R., Casella, L. G., Jones, J. W., Adams, M. D., Zurawski, D. V., Hazlett, K. R., et al. (2013). Unique structural modifications are present in the lipopolysaccharide from colistin-resistant strains of Acinetobacter baumannii. Antimicrob. Agents Chemother. 57, 4831-4840. doi: 10.1128/AAC.00865-13

Poirel, L., Jayol, A., Bontron, S., Villegas, M.-V., Ozdamar, M., Türkoglu, S., et al. (2014). The mgrB gene as a key target for acquired resistance to colistin in Klebsiella pneumoniae. J. Antimicrob. Chemother. doi: 10.1093/jac/dku323. [Epub ahead of print].

Pournaras, S., Poulou, A., Dafopoulou, K., Chabane, Y. N., Kristo, I., Makris, D., et al. (2014). Growth retardation, reduced invasiveness, and impaired colistin-mediated cell death associated with colistin resistance development in Acinetobacter baumannii. Antimicrob. Agents Chemother. 58, 828-832. doi: 10.1128/AAC.01439-13

Quesada, A., Porrero, M. C., Téllez, S., Palomo, G., García, M., and Domínguez, L. (2014). Polymorphism of genes encoding PmrAB in colistin-resistant strains of Escherichia coli and Salmonella enterica isolated from poultry and swine. J. Antimicrob. Chemother. doi: 10.1093/jac/dku320. [Epub ahead of print].

Raetz, C. R., Reynolds, C. M., Trent, M. S., and Bishop, R. E. (2007). Lipid A modification systems in gram-negative bacteria. Annu. Rev. Biochem. 76, 295-329. doi: 10.1146/annurev.biochem.76.010307.145803

Reynolds, C. M., Kalb, S. R., Cotter, R. J., and Raetz, C. R. (2005). A phosphoethanolamine transferase specific for the outer 3-deoxy-D-manno-octulosonic acid residue of Escherichia coli lipopolysaccharide. Identification of the eptB gene and $\mathrm{Ca}^{2+}$ hypersensitivity of an eptB deletion mutant. J. Biol. Chem. 280, 21202-21211. doi: 10.1074/jbc.M500964200

Reynolds, C. M., Ribeiro, A. A., McGrath, S. C., Cotter, R. J., Raetz, C. R., and Trent, M. S. (2006). An outer membrane enzyme encoded by Salmonella typhimurium lpxR that removes the 3'-acyloxyacyl moiety of lipid A. J. Biol. Chem. 281, 21974-21987. doi: 10.1074/jbc.M603527200

Rolain, J. M., Diene, S. M., Kempf, M., Gimenez, G., Robert, C., and Raoult, D. (2013). Real-time sequencing to decipher the molecular mechanism of resistance of a clinical pan-drug-resistant Acinetobacter baumannii isolate from Marseille, France. Antimicrob. Agents Chemother. 57, 592-596. doi: 10.1128/AAC.01314-12

Rolain, J. M., Roch, A., Castanier, M., Papazian, L., and Raoult, D. (2011). Acinetobacter baumannii resistant to colistin with impaired virulence: a case report from France. J. Infect. Dis. 204, 1146-1147. doi: 10.1093/infdis/ jir475

Roland, K. L., Martin, L. E., Esther, C. R., and Spitznagel, J. K. (1993). Spontaneous pmrA mutants of Salmonella typhimurium LT2 define a new two-component regulatory system with a possible role in virulence. J. Bacteriol. 175, 4154-4164.

Rozalski, A., Sidorczyk, Z., and Kotelko, K. (1997). Potential virulence factors of Proteus bacilli. Microbiol. Mol. Biol. Rev. 61, 65-89.

Samonis, G., Korbila, I. P., Maraki, S., Michailidou, I., Vardakas, K. Z., Kofteridis, D., et al. (2014). Trends of isolation of intrinsically resistant to colistin Enterobacteriaceae and association with colistin use in a tertiary hospital. Eur. J. Clin. Microbiol Infect. Dis. 33, 1505-1510. doi: 10.1007/s10096-014-2097-8

Sassera, D., Comandatore, F., Gaibani, P., D’Auria, G., Mariconti, M., Landini, M. P., et al. (2013). Comparative genomics of closely related strains of Klebsiella pneumoniae reveals genes possibly involved in colistin resistance. Ann. Microbiol. 64, 887-890. doi: 10.1007/s13213-013-0727-5

Schurek, K. N., Sampaio, J. L., Kiffer, C. R., Sinto, S., Mendes, C. M., and Hancock, R. E. (2009). Involvement of pmrAB and phoPQ in polymyxin B adaptation and inducible resistance in non-cystic fibrosis clinical isolates of Pseudomonas aeruginosa. Antimicrob. Agents Chemother. 53, 4345-4351. doi: 10.1128/AAC.01267-08

Sidorczyk, Z., Zahringer, U., and Rietschel, E. T. (1983). Chemical structure of the lipid A component of the lipopolysaccharide from a Proteus mirabilis Re-mutant. Eur. J. Biochem. 137, 15-22. doi: 10.1111/j.1432-1033.1983. tb07789.x

Silipo, A., Molinaro, A., Cescutti, P., Bedini, E., Rizzo, R., Parrilli, M., et al. (2005). Complete structural characterization of the lipid A fraction of a clinical strain of B. cepacia genomovar I lipopolysaccharide. Glycobiology 15, 561-570. doi: 10.1093/glycob/cwi029

Snitkin, E. S., Zelazny, A. M., Gupta, J., Palmore, T. N., Murray, P. R., and Segre, J. A. (2013). Genomic insights into the fate of colistin resistance and Acinetobacter baumannii during patient treatment. Genome Res. 23, 1155-1162. doi: 10.1101/gr.154328.112

Snitkin, E. S., Zelazny, A. M., Thomas, P. J., Stock, F., Henderson, D. K., Palmore, T. N., et al. (2012). Tracking a hospital outbreak of carbapenem-resistant Klebsiella pneumoniae with whole-genome sequencing. Sci. Transl. Med. 4, 148ra116. doi: 10.1126/scitranslmed.3004129

Srinivasan, V. B., and Rajamohan, G. (2013). KpnEF, a new member of the Klebsiella pneumoniae cell envelope stress response regulon, is an SMR-type efflux pump involved in broad-spectrum antimicrobial resistance. Antimicrob. Agents Chemother. 57, 4449-4462. doi: 10.1128/AAC.02284-12

Stein, A., and Raoult, D. (2002). Colistin: an antimicrobial for the 21st century? Clin. Infect. Dis. 35, 901-902. doi: 10.1086/342570

Sun, S., Negrea, A., Rhen, M., and Andersson, D. I. (2009). Genetic analysis of colistin resistance in Salmonella enterica serovar Typhimurium. Antimicrob. Agents Chemother. 53, 2298-2305. doi: 10.1128/AAC.01016-08

Tamayo, R., Choudhury, B., Septer, A., Merighi, M., Carlson, R., and Gunn, J. S. (2005a). Identification of cptA, a PmrA-regulated locus required for phosphoethanolamine modification of the Salmonella enterica serovar 
typhimurium lipopolysaccharide core. J. Bacteriol. 187, 3391-3399. doi: 10.1128/JB.187.10.3391-3399.2005

Tamayo, R., Prouty, A. M., and Gunn, J. S. (2005b). Identification and functional analysis of Salmonella enterica serovar Typhimurium PmrA-regulated genes. FEMS Immunol. Med. Microbiol. 43, 249-258. doi: 10.1016/j.femsim. 2004.08.007

Touze, T., Tran, A. X., Hankins, J. V., Mengin-Lecreulx, D., and Trent, M. S. (2008), Periplasmic phosphorylation of lipid A is linked to the synthesis of undecaprenyl phosphate. Mol. Microbiol. 67, 264-277. doi: 10.1111/j.1365-2958.2007.06044.x

Tran, A. X., Lester, M. E., Stead, C. M., Raetz, C. R., Maskell, D. J., McGrath, S. C., et al. (2005). Resistance to the antimicrobial peptide polymyxin requires myristoylation of Escherichia coli and Salmonella typhimurium lipid A. J. Biol. Chem. 280, 28186-28194. doi: 10.1074/jbc.M505020200

Trent, M. S., Pabich, W., Raetz, C. R., and Miller, S. I. (2001a). A PhoP/PhoQinduced Lipase (PagL) that catalyzes 3-O-deacylation of lipid A precursors in membranes of Salmonella typhimurium. J. Biol. Chem. 276, 9083-9092. doi: 10.1074/jbc.M010730200

Trent, M. S., Ribeiro, A. A., Lin, S., Cotter, R. J., and Raetz, C. R. (2001b). An inner membrane enzyme in Salmonella and Escherichia coli that transfers 4-amino4-deoxy-L-arabinose to lipid A: induction on polymyxin-resistant mutants and role of a novel lipid-linked donor. J. Biol. Chem. 276, 43122-43131. doi: 10.1074/jbc.M106961200

Vaara, M., Vaara, T., Jensen, M., Helander, I., Nurminen, M., Rietschel, E. T., et al. (1981). Characterization of the lipopolysaccharide from the polymyxinresistant pmrA mutants of Salmonella typhimurium. FEBS Lett. 129, 145-149. doi: 10.1016/0014-5793(81)80777-6

Velkov, T., Deris, Z. Z., Huang, J. X., Azad, M. A. K., Butler, M., Sivanesan, S., et al. (2013a). Surface changes and polymyxin interactions with a resistant strain of Klebsiella pneumoniae. Innate Immun. 20, 350-363. doi: 10.1177/1753425913 493337

Velkov, T., Soon, R. L., Chong, P. L., Huang, J. X., Cooper, M. A., Azad, M. A., et al. (2013b). Molecular basis for the increased polymyxin susceptibility of Klebsiella pneumoniae strains with under-acylated lipid A. Innate Immun. 19, 265-277. doi: $10.1177 / 1753425912459092$

Vinogradov, E., Lindner, B., Seltmann, G., Radziejewska-Lebrecht, J., and Holst, O. (2006). Lipopolysaccharides from Serratia marcescens possess one or two 4amino-4-deoxy-L-arabinopyranose 1-phosphate residues in the lipid A and Dglycero-D-talo-oct-2-ulopyranosonic acid in the inner core region. Chemistry 12, 6692-6700. doi: 10.1002/chem.200600186

Wang, W. B., Chen, I. C., Jiang, S. S., Chen, H. R., Hsu, C. Y., Hsueh, P. R., et al. (2008). Role of RppA in the regulation of polymyxin b susceptibility, swarming, and virulence factor expression in Proteus mirabilis. Infect. Immun. 76, 2051-2062. doi: 10.1128/IAI.01557-07
Weiss, J., Victor, M., Cross, A. S., and Elsbach, P. (1982). Sensitivity of K1-encapsulated Escherichia coli to killing by the bactericidal/permeabilityincreasing protein of rabbit and human neutrophils. Infect. Immun. 38, $1149-1153$.

Winfield, M. D., and Groisman, E. A. (2004). Phenotypic differences between Salmonella and Escherichia coli resulting from the disparate regulation of homologous genes. Proc. Natl. Acad. Sci. U.S.A. 101, 17162-17167. doi: 10.1073/pnas.0406038101

Wosten, M. M., and Groisman, E. A. (1999). Molecular characterization of the PmrA regulon. J. Biol. Chem. 274, 27185-27190. doi: 10.1074/jbc.274.38.27185

Yan, A., Guan, Z., and Raetz, C. R. (2007). An undecaprenyl phosphateaminoarabinose flippase required for polymyxin resistance in Escherichia coli. J. Biol. Chem. 282, 36077-36089. doi: 10.1074/jbc.M706172200

Yethon, J. A., Gunn, J. S., Ernst, R. K., Miller, S. I., Laroche, L., Malo, D., et al. (2000). Salmonella enterica serovar typhimurium waaP mutants show increased susceptibility to polymyxin and loss of virulence In vivo. Infect. Immun. 68, 4485-4491. doi: 10.1128/IAI.68.8.4485-4491.2000

Young, M. L., Bains, M., Bell, A., and Hancock, R. E. (1992). Role of Pseudomonas aeruginosa outer membrane protein $\mathrm{OprH}$ in polymyxin and gentamicin resistance: isolation of an OprH-deficient mutant by gene replacement techniques. Antimicrob. Agents Chemother. 36, 2566-2568. doi: 10.1128/AAC.36.11.2566

Zhou, Z., Ribeiro, A. A., Lin, S., Cotter, R. J., Miller, S. I., and Raetz, C. R. (2001). Lipid A modifications in polymyxin-resistant Salmonella typhimurium: PMRA-dependent 4-amino-4-deoxy-L-arabinose, and phosphoethanolamine incorporation. J. Biol. Chem. 276, 43111-43121. doi: 10.1074/jbc.M106960200

Conflict of Interest Statement: The authors declare that the research was conducted in the absence of any commercial or financial relationships that could be construed as a potential conflict of interest.

Received: 20 August 2014; accepted: 07 November 2014; published online: 26 November 2014.

Citation: Olaitan AO, Morand S and Rolain J-M (2014) Mechanisms of polymyxin resistance: acquired and intrinsic resistance in bacteria. Front. Microbiol. 5:643. doi: 10.3389/fmicb.2014.00643

This article was submitted to Antimicrobials, Resistance and Chemotherapy, a section of the journal Frontiers in Microbiology.

Copyright (๑) 2014 Olaitan, Morand and Rolain. This is an open-access article distributed under the terms of the Creative Commons Attribution License (CC BY). The use, distribution or reproduction in other forums is permitted, provided the original author(s) or licensor are credited and that the original publication in this journal is cited, in accordance with accepted academic practice. No use, distribution or reproduction is permitted which does not comply with these terms. 